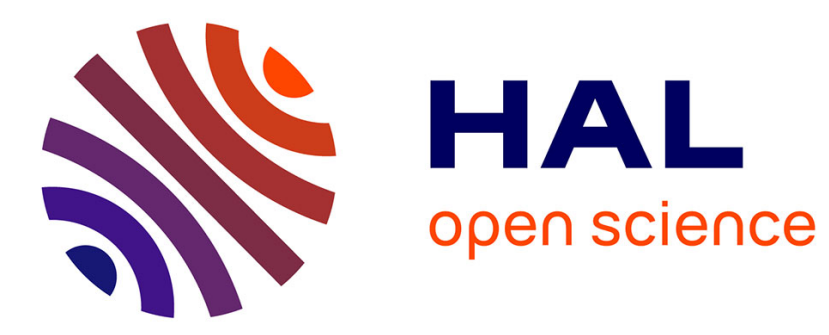

\title{
Evolution of granular media under constant-volume multidirectional cyclic shearing
}

Ming Yang, Mahdi Taiebat, Patrick Mutabaruka, Farhang Radjai

\section{To cite this version:}

Ming Yang, Mahdi Taiebat, Patrick Mutabaruka, Farhang Radjai. Evolution of granular media under constant-volume multidirectional cyclic shearing. Acta Geotechnica, 2021, 10.1007/s11440-021-012390 . hal-03344142

\author{
HAL Id: hal-03344142 \\ https://hal.science/hal-03344142
}

Submitted on 14 Sep 2021

HAL is a multi-disciplinary open access archive for the deposit and dissemination of scientific research documents, whether they are published or not. The documents may come from teaching and research institutions in France or abroad, or from public or private research centers.
L'archive ouverte pluridisciplinaire HAL, est destinée au dépôt et à la diffusion de documents scientifiques de niveau recherche, publiés ou non, émanant des établissements d'enseignement et de recherche français ou étrangers, des laboratoires publics ou privés. 


\title{
Evolution of granular media under constant-volume multidirectional cyclic shearing
}

\author{
Ming Yang ${ }^{1} \cdot$ Mahdi Taiebat $^{1}$ (D) $\cdot$ Patrick Mutabaruka $^{3} \cdot$ Farhang Radjai $^{2}$
}

\begin{abstract}
By means of the three-dimensional discrete element method, we study the long-time evolution toward liquefaction state in granular materials composed of spherical particles under multidirectional cyclic shearing at constant volume. Extensive simulations were carried out along 1-D linear, 2-D linear, circular/oval, and 8-like shear paths, and the evolution of the system was analyzed in terms of pore pressure, shear strain, and granular texture. The macroscopic stress path and stressstrain response agree well with laboratory experiments. We find that the liquefaction resistance, i.e., the number of cycles necessary to reach the liquefaction state, is generally lower under multidirectional loading as compared to unidirectional loading. As the transient vanishing of mean stress does not occur for all stress paths, we introduce a shear strain-based liquefaction criterion that can be consistently applied to all strain paths. The granular texture is monitored through the coordination number, particle connectivity, force and fabric anisotropies, and friction mobilization. In particular, a particlevoid descriptor, named centroid distance, is found to be closely related to the shear strain accumulation. We show that the force anisotropy tensors become almost proportional to the deviatoric stress tensor more quickly than the fabric anisotropy tensor, which takes most of the pre-liquefaction period to follow the external loading. The relationship between deviatoric stress ratio and the force and fabric anisotropies, known to hold in monotonic triaxial loading, also holds with high accuracy in the studied multidirectional cyclic shearing paths; the contributing weights of the anisotropies level off in the post-liquefaction period and do not depend on the shear path.
\end{abstract}

Keywords Anisotropy · DEM · Deformation · Granular materials · Liquefaction · Multidirectional shear · Stability

\section{Introduction}

The most common practice in conducting the dynamic analysis of geo-structures, from simpler cases of free field

Mahdi Taiebat

mtaiebat@civil.ubc.ca

Ming Yang

yangm15@civil.ubc.ca

Patrick Mutabaruka

patrick.mutabaruka@ifremer.fr

Farhang Radjaï

franck.radjai@umoutpellier.fr

1 Department of Civil Engineering, University of British Columbia, Vancouver, Canada

2 CNRS-University of Montpellier, LMGC, Montpellier, France

3 IFREMER, DYNECO/DHYSED, Plouzané, France site response analysis to more complex cases of soilstructure interactions, is using only a horizontal component of the ground motion. However, in real earthquakes, soil deposits are subjected to multidirectional cyclic shearing, and each component has variable amplitudes and many frequencies. More specifically, in these multidirectional cyclic shearing scenarios, in addition to a vertical component of the seismic loading, there are two horizontal shear components. Neglecting their simultaneous effects can potentially lead to an underestimation of seismic risk.

Pyke et al. [50] were the first to study sand response under multidirectional shaking, using shaking table tests on dry Monterey No. 0 sand. They found that the settlement 
caused by shaking in two horizontal directions was nearly the sum of the settlements caused by each component alone. Su and $\mathrm{Li}$ [61] conducted a pair of centrifuge tests on loose Toyoura sand under uni- and biaxial shaking, and the results indicated that the latter could develop $20 \%$ greater peak pore pressure near the sample bottom and $12 \%$ higher permanent settlement. Recently, El Shafee et al. [14] presented a series of centrifuge tests on a level site consisting of loose saturated Nevada 120 sand subjected to uni- and multidirectional base excitations. They concluded that the practice of only increasing the unidirectional shaking component by $10 \%$ actually underestimates the soil response under multidirectional shaking. Based on dynamic centrifuge tests on saturated dense Ottawa sand under uni- and multidirectional shaking, Cerna-Diaz et al. [10] also found that multidirectional shaking caused an increase in pore pressure and volumetric strain by approximately a factor two compared to unidirectional shaking. This is a considerably larger effect than found on loose sands by others.

To mimic the response of soil element in a level or sloping ground to idealized multidirectional cyclic shearing under undrained conditions, extensive laboratory experimental work has been conducted over the past decades $[6,26,28,39,62]$, generating a comprehensive experimental database for understanding the physics of the sheared system. The experimental findings show that the multidirectional cyclic shearing induces a considerable reduction in the liquefaction resistance compared to the unidirectional one with a similar shearing amplitude. All these laboratory findings are still on the overall macroscopic behavior given the minimal use of special experimental techniques in cyclic shearing, such as X-ray computed tomography [20] and photoelasticity technique [37]. The underlying mechanisms at the particle scale remain, to a large extent, to be explored and understood. In addition to experiments, continuum modeling of soil deposits under multidirectional shaking has been carried out by Ghaboussi and Dikmen [17], Su and Li [61], Carlton and Kaynia [9], Zeghal et al. [79], Yang et al. [77], and Reyes et al. [54], to name a few. The underlying constitutive models are not based on the particle-scale evolution of the granular microstructure under complex multidirectional shearing, which, to our best knowledge, remains essentially unexplored.

Discrete element method (DEM) [12] provides a suitable framework to study the mechanical response of granular materials from both macroscopic and grain-scale perspectives when subjected to multidirectional cyclic shearing. It also has the advantage of providing perfect reproducibility of each "numerical experiment" as the same sample can be reused, thereby eliminating random noise and natural uncertainty due to the variability of laboratory tests. Applying the DEM to reproduce liquefaction phenomenon induced by conventional unidirectional cyclic shearing such as triaxial or simple shear tests dates back to Ng and Dobry [44]. Later, Sitharam [58] explored the micromechanics to some extent by considering the drop of coordination number for loose samples approaching liquefaction. Huang et al. [22, 23] and Martin et al. [38] adopted another microscopic indicator called redundancy index to distinguish the liquefaction state from the non-liquefaction one; meanwhile, by extracting the mesoscale structures like loop-like clusters encompassed by contacts, they observed that the maximum cluster size decreases to very small values in the liquefaction state. Wang and Wei [68] and Wang et al. [69] came up with the centroid distance and mean neighboring particle distance, respectively, to explore the potential microscopic indicators influencing the post-liquefaction large deformation. Other topics of special interest for undrained cyclic shearing include, but are not limited to, fabric anisotropy evolution [53, 60, 72] and effects of initial and loading conditions on liquefaction resistance $[16,41,70,74,80]$, to name a few. Recently, Wei [71] and Wei et al. [73] conducted DEM simulations of a granular packing subjected to uni- and multidirectional cyclic shearing. They found that the same "stable fabric" was reached after a sufficient number of loading cycles regardless of multidirectional loading paths.

This paper introduces a novel presentation of the longterm evolution of granular microstructure in the discrete element numerical study of multidirectional cyclic shearing at constant volume. The focus is on the gradual evolution of the granular material and the onset of liquefaction from both macroscopic and particle-scale perspectives. A series of multidirectional cyclic tests along 1-D linear, 2-D linear, circular/oval, and 8-like shear paths are performed on a medium dense sample. We explore in detail the effect of shear paths on pore pressure generation and shear strain development. Four representative simulations are then selected for micromechanical investigation with three different approaches: (1) adopting contact-based descriptors to analyze the degradation of the overall stability of the contact network [3, 63]; (2) using void-based fabric to shed light on the post-liquefaction deformation [68]; (3) applying stress-force-fabric relationship to quantify the respective contributions of the fabric and force anisotropies to the load-bearing network [48]. 


\section{Numerical procedures}

\subsection{Multidirectional cyclic shear test}

Cyclic simple shear or torsional tests are usually carried out in the laboratory using harmonic shearing. Multidirectional cyclic shear test is similar to the simple shear test with the major difference that two horizontal shear components are simultaneously applied on the top of the soil specimen. The resultant shear stress can change in both magnitude and orientation with time. Generally, there are two stages in this test: a consolidation stage by applying confinement with or without static shear stress to bring the sample to a well-defined consolidated state, and an undrained cyclic shear stage by applying the cyclic shear stresses. The two components of shear stress, $\tau_{x}$ and $\tau_{y}$, evolve with time as

$$
\begin{aligned}
\frac{\tau_{x}}{p_{0}} & =\mathrm{SSR}_{x}+\mathrm{CSR}_{x} \sin \left(2 \pi f_{x} t\right), \\
\frac{\tau_{y}}{p_{0}} & =\mathrm{SSR}_{y}+\mathrm{CSR}_{y} \sin \left(2 \pi f_{y} t+\phi\right) .
\end{aligned}
$$

Here $p_{0}$ is the initial mean stress used for normalizing the shear stress, usually adopted for isotropically consolidated samples. For $\mathrm{K}_{0}$ consolidated samples that are prepared by unidirectional compression with lateral normal strains being constrained, $p_{0}$ is replaced by the initial vertical stress. Similar to the standard terminology as in simple shear tests, the static stress ratio (SSR) represents the ratio of static shear stress and initial confinement stress, and the cyclic stress ratio (CSR) represents the ratio of cyclic shear stress amplitude and initial confinement stress. Nonzero SSR mimics the initial stress state of a soil element under the sloping ground. One may expect two SSRs for two static shear stresses and two CSRs for two cyclic shear stresses in multidirectional cyclic shear test, but by a proper choice of the coordinate system, the nonzero component of SSR can be either $\mathrm{SSR}_{x}$ or $\mathrm{SSR}_{y} . f_{x}$ and $f_{y}$ are the frequencies of the two harmonic cyclic shear stresses along the $x$ and $y$ directions, respectively, and $\phi$ is the initial phase difference between the two cyclic components.

Given that the two cyclic stresses can have different amplitudes, frequencies, and phase angles, a wide range of different shear paths can be generated. In this paper, we consider four types of shear paths: 1-D linear, 2-D linear, circular/oval, and 8-like, as depicted in Fig. 1. The 1-D linear path is simply the traditional cyclic simple shear test, which may also include static or bias shear stress in the same direction as the subsequent undrained cyclic shearing. It can be attained by setting $\mathrm{SSR}_{x}=0, \mathrm{CSR}_{x}=0$ in Eq. (1) and $\phi=0$ in Eq. (2). The 1-D linear path indicates plane strain condition, mimicking unidirectional shaking of level $\left(\mathrm{SSR}_{x}=0\right)$ or sloping $\left(\mathrm{SSR}_{x} \neq 0\right)$ ground where shaking is parallel to the slope direction. When the unidirectional cyclic shearing is applied perpendicular to the direction of static shear stress, the shear path is a 2-D linear path, as shown in Fig. 1b. It can be achieved by setting $\mathrm{CSR}_{x}=0$ in Eq. (1) and $\operatorname{SSR}_{y}=0, \phi=0$ in Eq. (2). Circular/oval path is obtained by setting $\operatorname{SSR}_{x}=0, f_{x}=f_{y}$ and $\phi=\pi / 2$. When $\operatorname{CSR}_{x}=\mathrm{CSR}_{y}$, it corresponds to a circular path. Otherwise, it is an oval path. The 8-like path is configured by setting $\mathrm{SSR}_{y}=0, \phi=0$, and $f_{x}=2 f_{y}$. The component $\mathrm{SSR}_{[]}$breaks the symmetry of all the above shear paths.

\subsection{Method, sample preparation, and shearing protocols}

An in-house three-dimensional (3D) particle dynamics DEM platform, named GRFlow3D [42], was used in this study. The granular assembly is composed of polydisperse spheres interacting via soft-particle laws. The contact interactions of spheres consist of normal elastic repulsion, tangential sliding, rolling, and torsion. As the particles are assumed to be nearly rigid, the local strain variable $\delta_{[]}$ should be defined from particle positions, and the corresponding force $\hat{f_{[]}}$can be calculated using linear springdashpot model:

$\hat{f_{[]}}=-k_{[]} \delta_{[]}-c_{[]} \dot{\delta}_{[]}$,

where $k_{[]}$is the spring stiffness, and $c_{[]}$is the viscous dashpot coefficient. The subscript placeholders can be for $n$ (normal contact), $t$ (tangential sliding), $r$ (rolling), or $o$ (torsion). Given the radii of two particles, $a_{i}$ and $a_{j}$, and their positions, $\boldsymbol{r}_{i}$ and $\boldsymbol{r}_{j}$, the normal elastic contact deflection $\delta_{n}$ along the normal direction is the overlap between the two particles, given by

$\delta_{n}=\left\|\boldsymbol{r}_{i}-\boldsymbol{r}_{j}\right\|-a_{i}-a_{j}$.

To exclude the non-realistic attractive force due to viscous damping at incipient separation between two particles, the normal force $f_{n}$ is represented by a ramp function $R\left(\hat{f_{n}}\right)$ where $R(x)=x$ if $x>0$ and $R(x)=0$ if $x \leq 0$. The interparticle forces and torques exist only when $\delta_{n}<0$. For the tangential force $\hat{f_{t}}$, the strain variable $\delta_{t}$ is the cumulative relative tangential displacement as long as the contact between two particles exists. The tangential force $f_{t}$ is equal to $\hat{f_{t}}$ if $\left|\hat{f_{t}}\right|<\mu_{t} f_{n}$, where $\mu_{t}$ is the coefficient of friction, and to $\operatorname{sgn}\left(\hat{f_{t}}\right) \mu_{t} f_{n}$ if $\left|\hat{f_{t}}\right|>\mu_{t} f_{n}$. Calculating the rolling and torsional forces (torques) is analogous to the tangential force. The strain variable $\delta_{r}$ for rolling is the cumulative relative rotation of two particles in the contact plane, whereas the strain variable $\delta_{o}$ for torsion is the cumulative relative rotation of two particles about their common axis (perpendicular to the contact plane). Their conjugate 


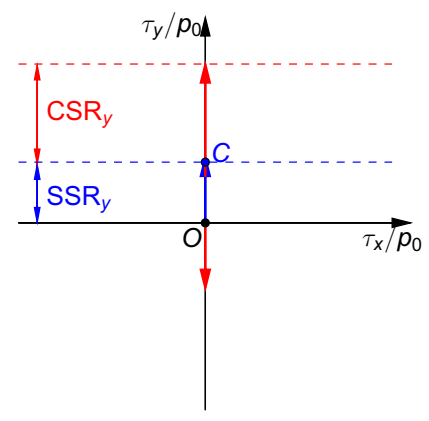

(a)

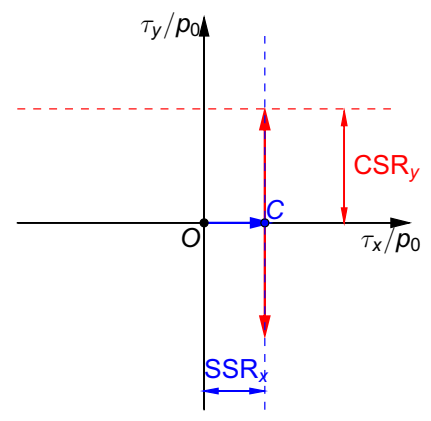

(b)

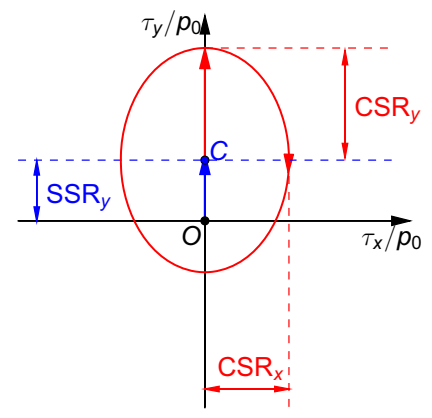

(c)

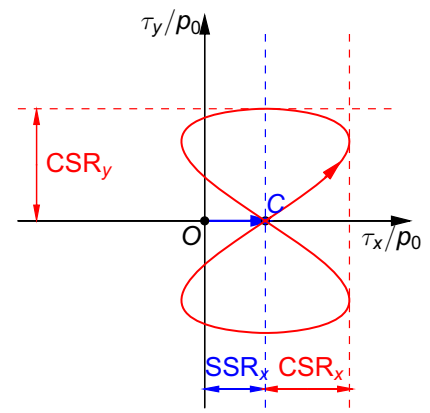

(d)

Fig. 1 Shear paths simulated in this study (modified from Yang et al. [78]): a 1-D linear shear path; b 2-D linear shear path; c circular/oval shear path; d 8-like shear path. The applied shear stress in the consolidation stage and the undrained cyclic shear stage are colored in blue and red, respectively (color figure online)

variables are $f_{r}$ and $f_{o}$, respectively, which both have the dimension of a torque. The rolling friction coefficient and torsion friction coefficient are defined as $f_{r}=\operatorname{sgn}\left(\hat{f}_{r}\right) \mu_{r} a f_{n}$ (for $\left|\hat{f_{r}}\right|>\mu_{r} a f_{n}$ ) and $f_{o}=\operatorname{sgn}\left(\hat{f_{o}}\right) \mu_{o} a f_{n}$ (for $\left|\hat{f}_{o}\right|>\mu_{o} a f_{n}$ ), respectively, where $a=a_{i}+a_{j}$. Unlike the normal deflection $\delta_{n}$, the other three elastic deflections cannot be directly calculated, but should be cumulated by integration over time from the instant two particles come to contact, as explained in detail in $[36,51]$.

Once all the forces and torques on a particle are obtained, the translational and angular accelerations can be calculated using Newton's second law of motion. These accelerations, together with the particle velocities at the beginning of each time step, are then used to update the velocities and positions of all particles. A velocity-Verlet time-stepping scheme was used in the simulations.

The simulations involve two stages: preparing a particle assembly via isotropic compression and applying a specified shear path to the assembly under the constant-volume condition. The sample consists of spheres with weak size plolydispersity, i.e., $d_{\max } / d_{\min }=2$ where $d_{\max }$ and $d_{\min }=$ $1.0 \mathrm{~mm}$ refer to the maximum and minimum particle diameters, respectively. Between $d_{\min }$ and $d_{\max }$, the particle volumes follow a uniform distribution. One can refer to $[43,67]$ for details of generating the particle size distribution. Once the particles with their sizes are generated, they are placed randomly on a 3D sparse lattice to avoid overlaps. This 3D lattice is contained in a rectangular cell whose top and bottom sides are rigid walls, and the four lateral sides are periodic boundaries, denoted as a bi-periodic cell.

The samples are compressed isotropically by moving the six sides of the cell. During the compression process, the gravity is set to zero. The six sides follow a translational motion. The tangential contact friction coefficient $\mu_{t}$ is tuned to achieve a certain value of the void ratio $e$, defined as the ratio of the total pore volume to the solid volume.
Many of the laboratory procedures for sample preparation at different densities cannot be precisely simulated; therefore, we adopted a simple computational procedure, modified from [34, 64], to prepare samples comparable with the laboratory ones. The procedure consists of four substages, which we describe here by taking the case of constructing a medium dense sample with the target mean stress $p_{0}=100 \mathrm{kPa}$ : (1) with $\mu_{t}=0.20$, densifying the sparse sample by moving the six sides at a constant speed until void ratio $e$ reaches 1.0 ; (2) setting velocities of particles and the six sides to zero, and using a servo-control algorithm to compress the sample isotropically with the target $p=10 \mathrm{kPa}$ where $\mu_{t}$ remains 0.2 ; (3) increasing the target $p$ to half of $p_{0}$, i.e., $50 \mathrm{kPa}$, and continuing compression of the sample with $\mu_{t}=0.20$; (4) modifying $\mu_{t}$ to 0.5 used for further compressing the sample with the target $p=p_{0}=$ $100 \mathrm{kPa}$ and subsequent cyclic shearing. One can refer to $[47,63]$ for the details of the servo-control algorithm. The first three substages generate an initially dense packing by controlling the tangential friction coefficient and increasing the confinement. The last substage is necessary to obtain a smooth distribution of $\left|f_{t}\right| /\left(\mu_{t} f_{n}\right)$ between 0 and 1 as usually a different $\mu_{t}$ is used in the stage of cyclic shearing. We conducted other simulations on samples with different numbers of spheres ranging between 2197 and 10,648. We did not observe significant improvement in the macroscopic response under constant-volume (isochoric) cyclic shearing for samples larger than 8000 spheres. Hence, samples of 8000 spheres were used in this study, falling into a range similar to that presented in $[34,38]$. Figure $2 \mathrm{a}$ displays one of the samples prepared by the above procedure. The void ratio in all simulations analyzed in this paper is $e=0.647$.

During cyclic shearing, the sample volume was kept constant. The constant-volume condition in cyclic shearing mimics the deformation of a porous solid matrix filled with an incompressible pore fluid without the drainage of the 


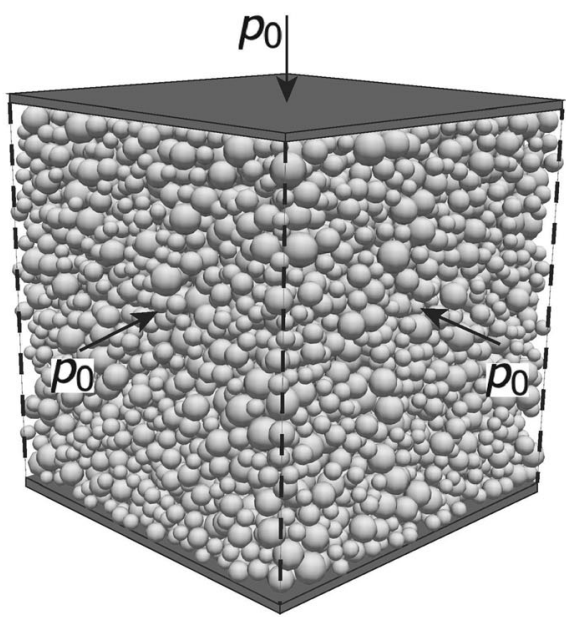

(a)

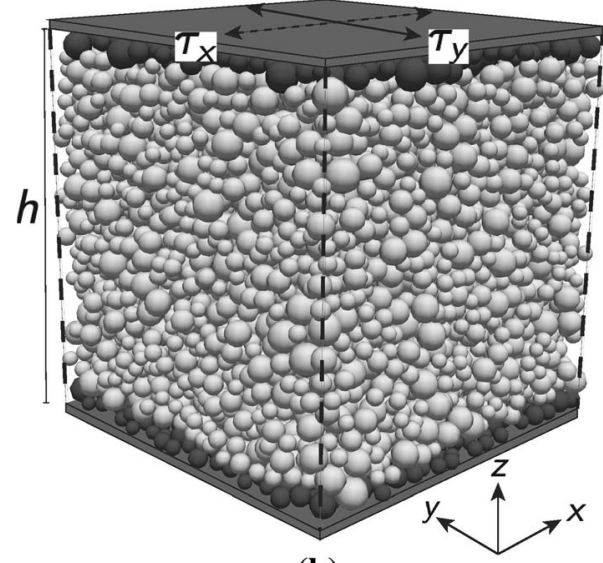

(b)

Fig. 2 Illustration of particle arrangements and boundary conditions for a sample composed of 8000 particles: a at the end of sample preparation; b during constant-volume cyclic shearing. The dark gray particles are glued to the top and bottom walls of the simulation cell

latter, i.e., a "truly undrained test." Both laboratory experiments and DEM studies have confirmed the similarity of the results in these two systems [5, 13]. The constant-volume condition was achieved by fixing four lateral sides and the bottom wall and keeping the sample height $h$ constant (see Fig. 2b). The top wall was free to move horizontally for the application of shear. The specified stress paths were exerted via a servo-controlled approach proposed by Wei et al. [73]. To reduce possible rigid slippage between the walls and mobile particles, a layer of particles was glued to the top and bottom walls as indicated by black spheres in Fig. $2 b$.

For the choice of the shear rate, the inertial number $I=\dot{\gamma} d \sqrt{\rho / p}$ was used, where $\dot{\gamma}=v / h$ is the shear strain rate with $v$ the horizontal velocity of the top wall, $\rho$ the density of particles, $d$ the mean diameter of particles, and $p$ the mean stress. The shear is considered to be quasistatic if
$I \ll 1$ [40], and the threshold is typically chosen as $10^{-3}$. Below this value, the behavior is practically independent of the shear rate. In constant-volume cyclic shearing, $I$ varies due to the change of $p$. When $p$ degrades in cyclic shearing and approaches vanishingly small values, $I$ may momentarily increase beyond $10^{-3}$. For a range of velocities $v$ of the top wall between $0.005 \mathrm{~m} / \mathrm{s}$ and $0.01 \mathrm{~m} / \mathrm{s}$ in the 1-D linear path with a zero $\operatorname{SSR}_{y}$, it was found that the macroscopic response even when $p$ is very small does not change noticeably during the whole shearing process. Even at such minimal values of $p, I$ does not exceed 0.05 (evidence provided in the next section). Therefore, in all the simulations reported in this paper, the velocity $v=$ $0.01 \mathrm{~m} / \mathrm{s}$ was adopted, corresponding to a shear strain rate $\dot{\gamma} \simeq 0.38 \mathrm{~s}^{-1}$ and consistent with [38]. For this choice, the simulations are faster, and at the same time, shearing is quasi-static when $p$ is not very small. The higher values of $I$ at very small $p$ arise from unstable deformation, which in our simulations is an intrinsic feature of cyclic liquefaction only and not influenced by the loading rate.

We checked the velocity profile of the sample during the cyclic shearing. Before $p$ drops to very small values, the velocity profile is almost linear across the sample, i.e., the whole sample is sheared by the motion of the top wall. When $p$ becomes tiny, the linear velocity profile disappears, and the whole system enters a chaotic regime with highly fluctuating velocity profiles.

The simulation parameters are given in Table 1. One can introduce the stiffness number $\kappa$ such that the average normal deflection $\delta_{n}$ between two particles in contact satisfies $\delta_{n} / d \propto \kappa^{-1}$ [51]. For the linear contact law in the normal direction, $\kappa=k_{n} /(p d)$. In this study, $k_{n}$ was set to $10^{6} \mathrm{~N} / \mathrm{m}$ to guarantee $\delta_{n} \sim 10^{-3} d$ in each contact, i.e., the

Table 1 DEM parameters

\begin{tabular}{ll}
\hline Description & Value \\
\hline Density, $\rho$ & $2650 \mathrm{~kg} / \mathrm{m}^{3}$ \\
Normal stiffness, $k_{n}$ & $10^{6} \mathrm{~N} / \mathrm{m}$ \\
Normal viscosity, $c_{n}$ & $1.15 \mathrm{~kg} / \mathrm{s}$ \\
Tangential stiffness, $k_{t}$ & $0.8 k_{n}$ \\
Tangential viscosity, $c_{t}$ & $0.2 c_{n}$ \\
Tangential friction coefficient, $\mu_{t}$ & $0.2^{+} \& 0.5^{*}$ \\
Rolling stiffness, $k_{r}$ & $0.1 k_{n}$ \\
Rolling viscosity, $c_{r}$ & $0.05 c_{n}$ \\
Rolling friction coefficient, $\mu_{r}$ & 0.1 \\
Torsion stiffness, $k_{o}$ & $0.1 k_{n}$ \\
Torsion viscosity, $c_{o}$ & $0.05 c_{n}$ \\
Torsion friction coefficient, $\mu_{o}$ & 0.1 \\
\hline
\end{tabular}

${ }^{+}$Isotropic compression stage ${ }^{*}$ cyclic shearing stage 
particles can be considered as nearly undeformable [43]. Then, $c_{n}$ is is fixed to get a value of 0.15 for the normal coefficient of restitution based on [57]. We also set $\mu_{t}=0.5$, which is a common value of the friction coefficient $[19,23,27]$. The values for other microscopic material parameters can be obtained from their relations to $k_{n}, c_{n}$ or $\mu_{t}$ as suggested by Luding [36], and they are listed in Table 1. The rolling and torsion stiffnesses and their corresponding friction coefficients are set to a small nonzero value in order to make rotations slightly dissipative and accounting in a simple way for the effects due to aspherical particle shape [15].

Ideally, for simulations of $\operatorname{SSR}_{[]} \neq 0$ with the subscript [] for $x$ or $y$, the static shear stress should be applied in the compression stage. In this study, however, $\mathrm{SSR}_{[]}$was applied in the constant-volume cyclic shearing stage just before adding the cyclic shear stress. As the current study focuses on the effect of shear paths on the granular assembly's cyclic response, it is natural to use the same initial sample. These two scenarios may be slightly different in practice. However, given that the sample prepared in this study is medium dense, the initial condition is not expected to change significantly if $\mathrm{SSR}_{[]}$is applied in the compression stage. The obvious impact of $\mathrm{SSR}_{[]}$on the sample response lies in its presence in the cyclic shearing stage, which shifts the cyclic shear paths along a certain direction. Furthermore, here we are mainly interested in the long-time evolution for which the memory of the initial preparation stage disappears along with cyclic shearing.

Table 2 lists 57 multidirectional cyclic shear tests simulated in this work, including the conditions implied by the second to sixth columns. The last three columns of Table 2 refer to the simulation outcomes, which will be explained in the sequel.

\section{Macroscopic response}

Stresses and strains at the sample scale can be computed in the cyclic shearing phase and used to follow the evolution of the mean effective stress and shear strain. The stress tensor $\boldsymbol{\sigma}$ is an average defined over a selected volume $V$ involving the inter-particle forces and contact normal orientations:

$\boldsymbol{\sigma}=\frac{1}{V} \sum_{c \in N_{c}} \boldsymbol{l}^{c} \otimes \boldsymbol{f}^{c}$

where $l^{c}$ is the branch vector connecting the centers of two particles for interior contacts or connecting the particle center and the contact point for exterior contacts, $f^{c}$ is the contact force, $\otimes$ denotes the tensor dyadic product and the summation runs over all the contacts $N_{c}$ in the selected
Table 2 Conditions and outcomes of multidirectional cyclic shear tests on a medium dense sample ${ }^{\dagger}$

\begin{tabular}{|c|c|c|c|c|c|c|c|c|}
\hline Test & $\begin{array}{l}\text { Shear } \\
\text { path }\end{array}$ & $\operatorname{SSR}_{x}$ & $\mathrm{SSR}_{y}$ & $\mathrm{CSR}_{x}$ & $\mathrm{CSR}_{y}$ & $N_{\mathrm{IL}}$ & $r_{u, \lim }$ & $\tau_{u, \lim } / p_{0}$ \\
\hline 1 & $\begin{array}{l}\text { 1-D } \\
\text { linear }\end{array}$ & & & & 0.20 & 65.3 & 1.00 & 0.00 \\
\hline 2 & & & 0.05 & & 0.20 & 53.3 & 1.00 & 0.00 \\
\hline 3 & & & 0.10 & & 0.20 & 35.2 & 1.00 & 0.00 \\
\hline 4 & & & 0.15 & & 0.20 & 24.2 & 1.00 & 0.00 \\
\hline 5 & & & 0.20 & & 0.20 & 37.2 & 1.00 & 0.00 \\
\hline 6 & & & 0.25 & & 0.20 & 70.2 & 0.64 & 0.05 \\
\hline 7 & & & 0.30 & & 0.20 & 117.2 & 0.42 & 0.11 \\
\hline 8 & & & & & 0.25 & 17.3 & 1.00 & 0.00 \\
\hline 9 & & & 0.05 & & 0.25 & 15.8 & 1.00 & 0.00 \\
\hline 10 & & & 0.10 & & 0.25 & 12.2 & 1.00 & 0.00 \\
\hline 11 & & & 0.20 & & 0.25 & 11.2 & 1.00 & 0.00 \\
\hline 12 & & & 0.30 & & 0.25 & 39.2 & 0.61 & 0.05 \\
\hline 13 & & & & & 0.30 & 8.7 & 1.00 & 0.00 \\
\hline 14 & & & 0.10 & & 0.30 & 7.2 & 1.00 & 0.00 \\
\hline 15 & & & 0.20 & & 0.30 & 7.2 & 1.00 & 0.00 \\
\hline 16 & & & 0.30 & & 0.30 & 18.2 & 1.00 & 0.00 \\
\hline 17 & & & 0.30 & & 0.35 & 6.2 & 1.00 & 0.00 \\
\hline 18 & $\begin{array}{l}\text { 2-D } \\
\text { linear }\end{array}$ & 0.05 & & & 0.20 & 60.6 & 0.91 & 0.05 \\
\hline 19 & & 0.10 & & & 0.20 & 42.0 & 0.80 & 0.10 \\
\hline 20 & & 0.15 & & & 0.20 & 26.6 & 0.69 & 0.15 \\
\hline 21 & & 0.20 & & & 0.20 & 21.7 & 0.56 & 0.20 \\
\hline 22 & & 0.30 & & & 0.20 & 54.7 & 0.22 & 0.30 \\
\hline 23 & & 0.05 & & & 0.25 & 15.1 & 0.91 & 0.05 \\
\hline 24 & & 0.10 & & & 0.25 & 12.1 & 0.80 & 0.10 \\
\hline 25 & & 0.20 & & & 0.25 & 9.2 & 0.55 & 0.20 \\
\hline 26 & & 0.30 & & & 0.25 & 15.1 & 0.26 & 0.31 \\
\hline 27 & & 0.05 & & & 0.30 & 6.5 & 0.91 & 0.05 \\
\hline 28 & & 0.10 & & & 0.30 & 6.0 & 0.79 & 0.10 \\
\hline 29 & & 0.20 & & & 0.30 & 5.5 & 0.57 & 0.20 \\
\hline 30 & & 0.30 & & & 0.30 & 7.1 & 0.27 & 0.31 \\
\hline 31 & $\begin{array}{c}\text { Circular/ } \\
\text { oval }\end{array}$ & & & 0.15 & 0.15 & 48.9 & 0.71 & 0.15 \\
\hline 32 & & & 0.10 & 0.15 & 0.15 & 29.6 & 0.91 & 0.05 \\
\hline 33 & & & 0.20 & 0.15 & 0.15 & 23.6 & 0.89 & 0.05 \\
\hline 34 & & & & 0.05 & 0.20 & 56.8 & 0.91 & 0.05 \\
\hline 35 & & & & 0.10 & 0.20 & 34.3 & 0.80 & 0.10 \\
\hline 36 & & & & 0.15 & 0.20 & 16.9 & 0.70 & 0.15 \\
\hline 37 & & & & 0.20 & 0.20 & 9.2 & 0.61 & 0.20 \\
\hline 38 & & & 0.05 & 0.20 & 0.20 & 8.5 & 0.70 & 0.15 \\
\hline 39 & & & 0.10 & 0.20 & 0.20 & 6.7 & 0.80 & 0.10 \\
\hline 40 & & & 0.15 & 0.20 & 0.20 & 5.6 & 0.91 & 0.05 \\
\hline 41 & & & 0.20 & 0.20 & 0.20 & 5.9 & 1.00 & 0.00 \\
\hline 42 & & & 0.25 & 0.20 & 0.20 & 8.7 & 0.90 & 0.05 \\
\hline 43 & & & 0.30 & 0.20 & 0.20 & 17.8 & 0.65 & 0.12 \\
\hline
\end{tabular}


Table 2 (continued)

\begin{tabular}{|c|c|c|c|c|c|c|c|c|}
\hline Test & $\begin{array}{l}\text { Shear } \\
\text { path }\end{array}$ & $\operatorname{SSR}_{x}$ & $\mathrm{SSR}_{y}$ & $\mathrm{CSR}_{x}$ & $\mathrm{CSR}_{y}$ & $N_{\mathrm{IL}}$ & $r_{u, \mathrm{lim}}$ & $\tau_{u, \lim } / p$ \\
\hline 44 & & & & 0.25 & 0.25 & 3.7 & 0.50 & 0.25 \\
\hline 45 & & & 0.30 & 0.25 & 0.25 & 4.8 & 0.90 & 0.05 \\
\hline 46 & 8-like & & & 0.15 & 0.15 & 21.1 & 1.00 & 0.00 \\
\hline 47 & & 0.10 & & 0.15 & 0.15 & 12.0 & 0.92 & 0.04 \\
\hline 48 & & 0.20 & & 0.15 & 0.15 & 16.0 & 0.76 & 0.11 \\
\hline 49 & & & & 0.05 & 0.20 & 44.5 & 1.00 & 0.00 \\
\hline 50 & & & & 0.10 & 0.20 & 20.1 & 1.00 & 0.00 \\
\hline 51 & & & & 0.15 & 0.20 & 9.8 & 1.00 & 0.00 \\
\hline 52 & & & & 0.20 & 0.20 & 5.5 & 1.00 & 0.00 \\
\hline 53 & & 0.10 & & 0.20 & 0.20 & 4.0 & 0.92 & 0.04 \\
\hline 54 & & 0.20 & & 0.20 & 0.20 & 4.8 & 0.81 & 0.09 \\
\hline 55 & & 0.30 & & 0.20 & 0.20 & 12.6 & 0.62 & 0.17 \\
\hline 56 & & & & 0.25 & 0.25 & 2.3 & 1.00 & 0.00 \\
\hline 57 & & 0.30 & & 0.25 & 0.25 & 4.5 & 0.67 & 0.15 \\
\hline
\end{tabular}

${ }^{\dagger}$ Void ratio $e=0.647$ and $p_{0}=100 \mathrm{kPa}$ measured at the end of isotropic compression

Empty space in the third, fourth, and fifth columns implies zero value

volume $V$. The superscript $c$ in $\boldsymbol{l}^{c}$ and $\boldsymbol{f}^{c}$ will be dropped in the sequel for simplicity. The two shear stresses $\tau_{x}$ and $\tau_{y}$ in Eqs. (1) and (2) refer to the components $\sigma_{z x}$ and $\sigma_{z y}$, respectively. The mean effective stress $p$ is given by $\left(\sigma_{x x}+\sigma_{y y}+\sigma_{z z}\right) / 3$.

Pore water is not explicitly included in the present DEM model; however, the deduced excess pore pressure in the "equivalent" undrained system with an incompressible pore fluid is computed as the variation of the simulated reduction in mean effective stress

$\Delta u=p_{0}-p$.

It is common to use the dimensionless pore pressure ratio defined by

$r_{u}=\frac{\Delta u}{p_{0}}=1-\frac{p}{p_{0}}$.

Vanishing pressure $p$ corresponds to $r_{u} \simeq 1$. From this point forward, any reference to pore pressure or pore pressure ratio simply means the deduced values from Eqs. (6) and (7).

The shear strain components $\left(\gamma_{x}, \gamma_{y}\right)$ are measured as

$\gamma_{x}=\frac{x_{w}}{h}, \gamma_{y}=\frac{y_{w}}{h}$

where $x_{w}$ and $y_{w}$ refer to the cumulative horizontal displacements of the top wall along $x$ and $y$ directions, respectively. Their values at any given time $t$ can be calculated as $x_{w}(t)=\int_{0}^{t} v_{x} \mathrm{~d} t, y_{w}(t)=\int_{0}^{t} v_{y} \mathrm{~d} t$

with $v_{x}$ and $v_{y}$ representing the velocities of the top wall in the $x$ and $y$ directions, respectively.

Before presenting the results of multidirectional cyclic shear tests, let us consider Fig. 3, which displays the evolution of inertial number for the constant-volume cyclic simple shear test with CSR $=0.20$ (test 1 in Table 2). We see that $I$ stays below $10^{-4}$ when $N<60$ and following the first liquefaction oscillates between $10^{-4}$ and 0.05 . The states with $I>10^{-3}$ reflect the small values of $p$ in the liquefaction state. Note that the mean stress $p$ never vanishes as otherwise $I$ would diverge. Hence, the liquefaction state corresponds here to the unstable collapse of the microstructure and its fragile and mobile nature, as described in the sequel.

Figure 4 compares the stress paths from the constantvolume monotonic and cyclic simple shear tests with $\mathrm{CSR}=0.20$. Both tests start from the same state of the initial packing (IP) and show a contraction tendency (decreasing $p$ ) at the beginning of shearing. Their stress paths overlap before the shear reversal in the cyclic test. The contraction tendency continues in the monotonic test until the phase transformation (PT) state. It then switches to dilation tendency (increasing $p$ ) until reaching the critical state (CS) presented in the inset window. The cyclic plot presents oscillations of stress path with a general decreasing trend of $p$ and finally gets trapped in a butterfly shape, referred to as "cyclic mobility". Two lines are drawn from the origin to the PT and CS of the monotonic test with slopes $M_{p}$ and $M_{c}$, respectively. A third line is determined to approximate the dilation phase of the butterfly shape with slope $M_{f}$. The phase transformation of the monotonic test appears to have a higher slope than that of the cyclic test. Also, the cyclic test does not reach the critical state in the dilation phase of the butterfly shape, given that

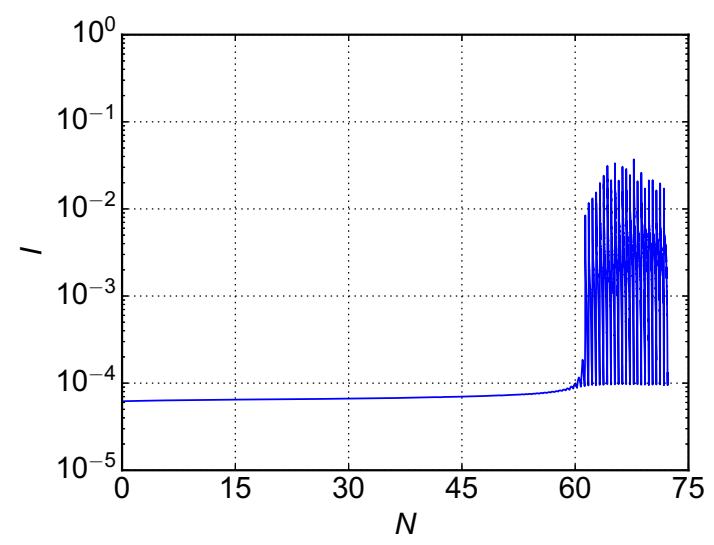

Fig. 3 Evolution of inertial number $I$ for the constant-volume cyclic simple shear test with CSR $=0.20$ 


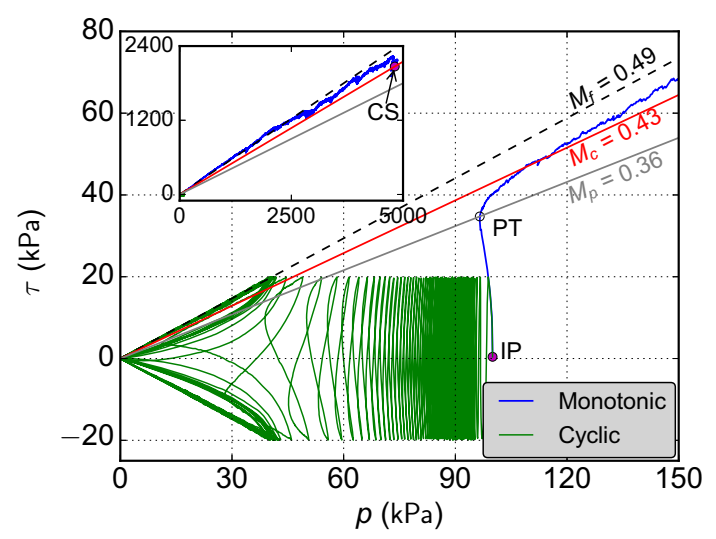

Fig. 4 Comparison of stress paths in constant-volume monotonic and cyclic simple shear tests $(C S R=0.20)$. IP stands for initial packing, PT for phase transformation, and CS for critical state. The straight lines represent the phase transformation (slope $M_{p}$ ) and critical state (slope $M_{c}$ ) based on monotonic test and dilation phase (slope $M_{f}$ ) based on cyclic test. The inset shows the monotonic shear path at large mean stress values

$M_{f}>M_{c}$. The states of IP and CS will be used later for further comparisons.

\subsection{4-way plot}

The macroscopic behavior of the granular sample under the four shear paths is displayed in Figs. 5, 6, 7, and 8, in the form of a 4-way plot [28]. These figures refer to the tests 1, 19, 37, and 52, respectively, in Table 2, all with the same CSR of 0.20 .

The 4-way plot for 1-D linear path consists of stress path, stress-strain loop, deduced pore pressure ratio evolution, and shear strain development. The 4-way plots for the other three paths consist of the applied shear stress path, the shear strain path, deduced pore pressure ratio evolution, and development of the resultant shear strain $\gamma \equiv \sqrt{\gamma_{x}^{2}+\gamma_{y}^{2}}$. Among the selected DEM simulations in these four figures, only the 2-D linear path has a static shear ratio of 0.10 . For each simulation, "initial liquefaction" is defined as when the resultant shear strain reaches $3.0 \%$ for the first time [28], i.e., $\gamma_{\mathrm{IL}}=3.0 \%$, and the corresponding number of cycles is denoted as $N_{\mathrm{IL}}$, as shown in Figs. 5, 6, 7 and 8. This state divides the cyclic shearing stage into two parts, pre-liquefaction and post-liquefaction, for the period prior to and after initial liquefaction, colored in gray and dark red in these figures, respectively. The limit pore pressure ratio $r_{u \text { lim }}$ is defined by the maximum achieved values of pore pressure ratio. The corresponding resultant shear stress and number of cycles are denoted as $\tau_{u, \text { lim }}$ and $N_{u, \text { lim }}$, respectively.

In these four simulations, one can see that notional pore pressure develops along with cyclic shearing. However, its limit value $r_{u, \text { lim }}$ reaches 1.0 in 1-D linear and 8-like paths
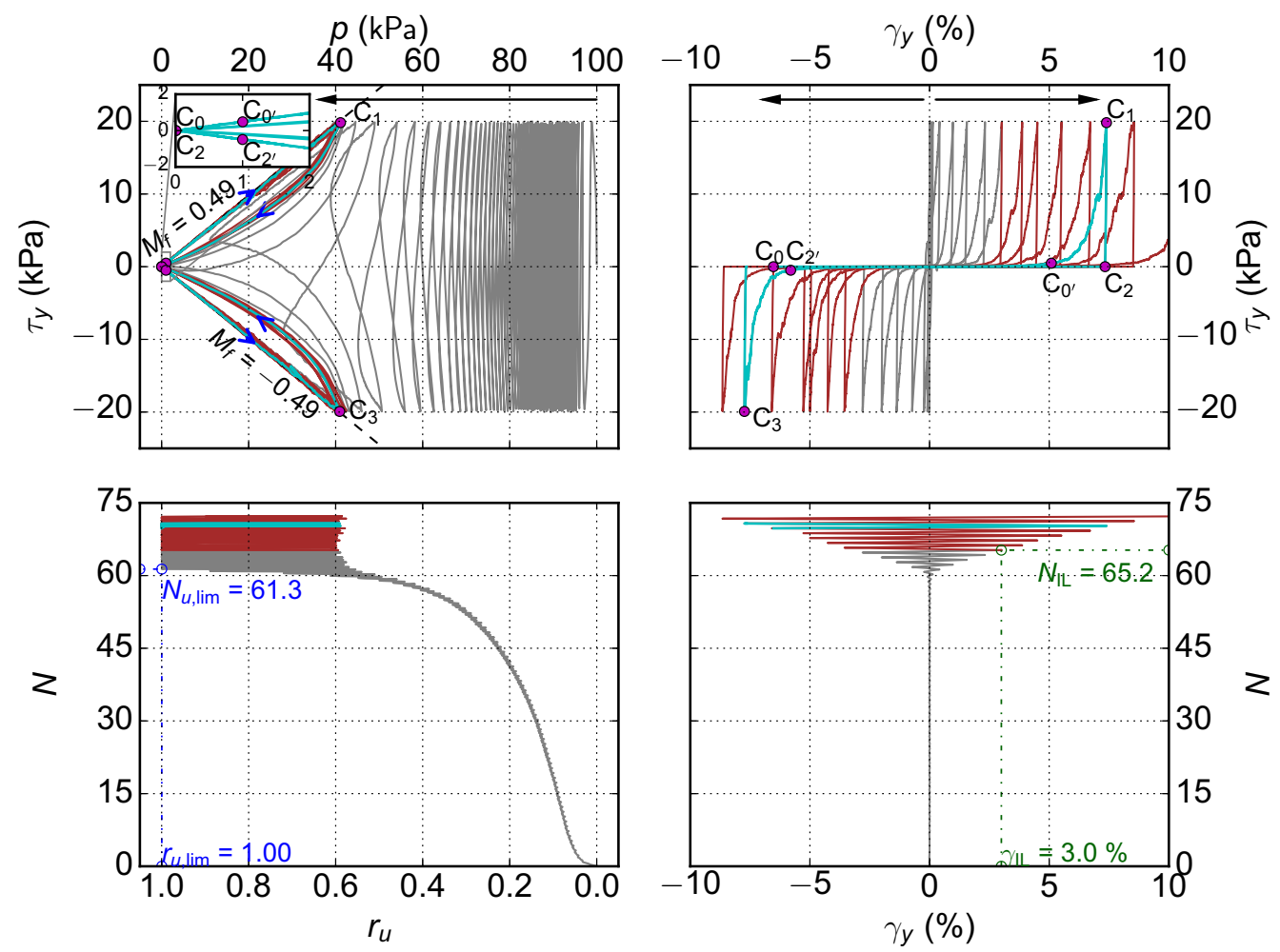

Fig. 5 Macroscopic response of constant-volume simple shear test with CSR $=0.20$ 

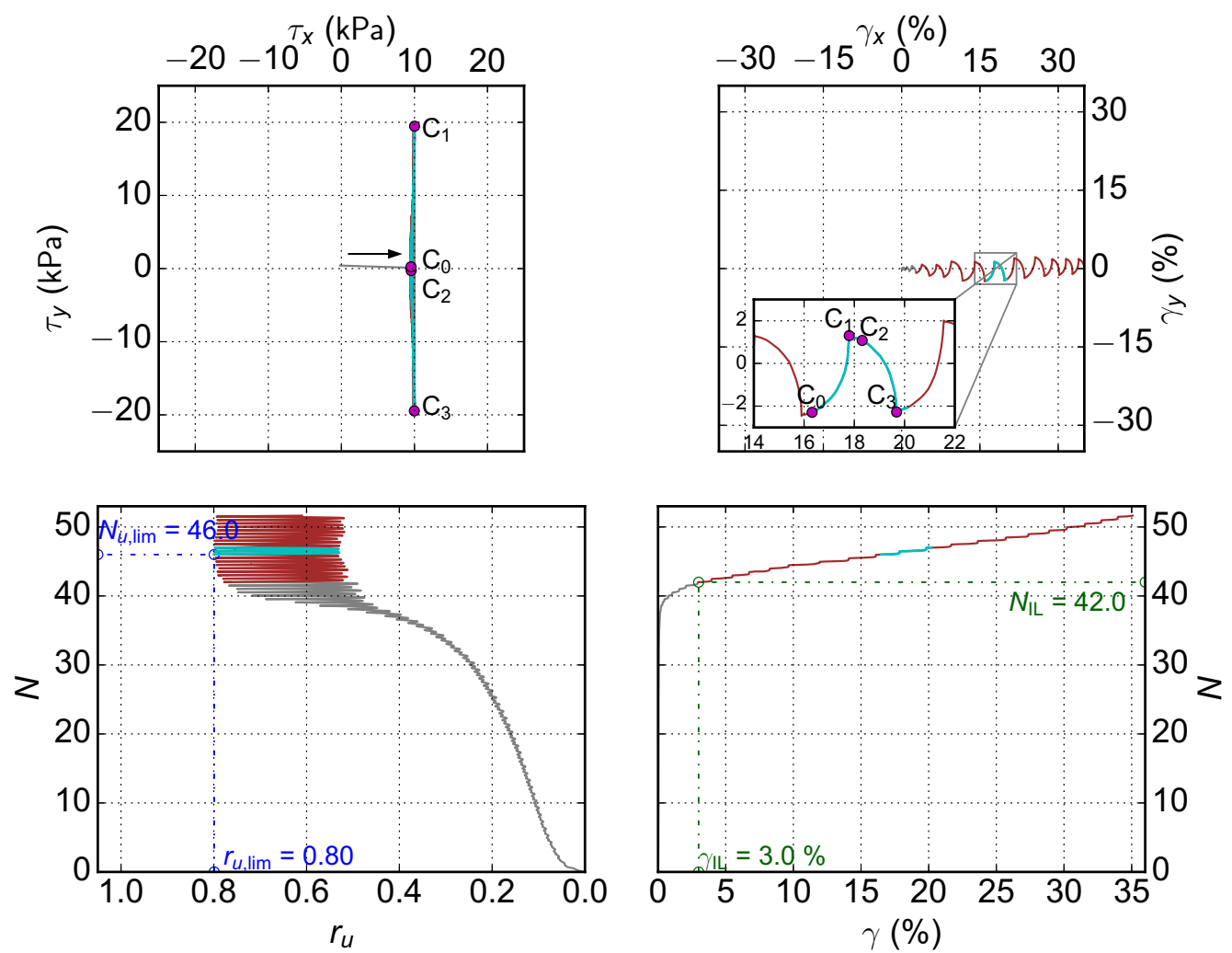

Fig. 6 Macroscopic response of constant-volume multidirectional cyclic shear test with CSR =0.20: 2-D linear shear path
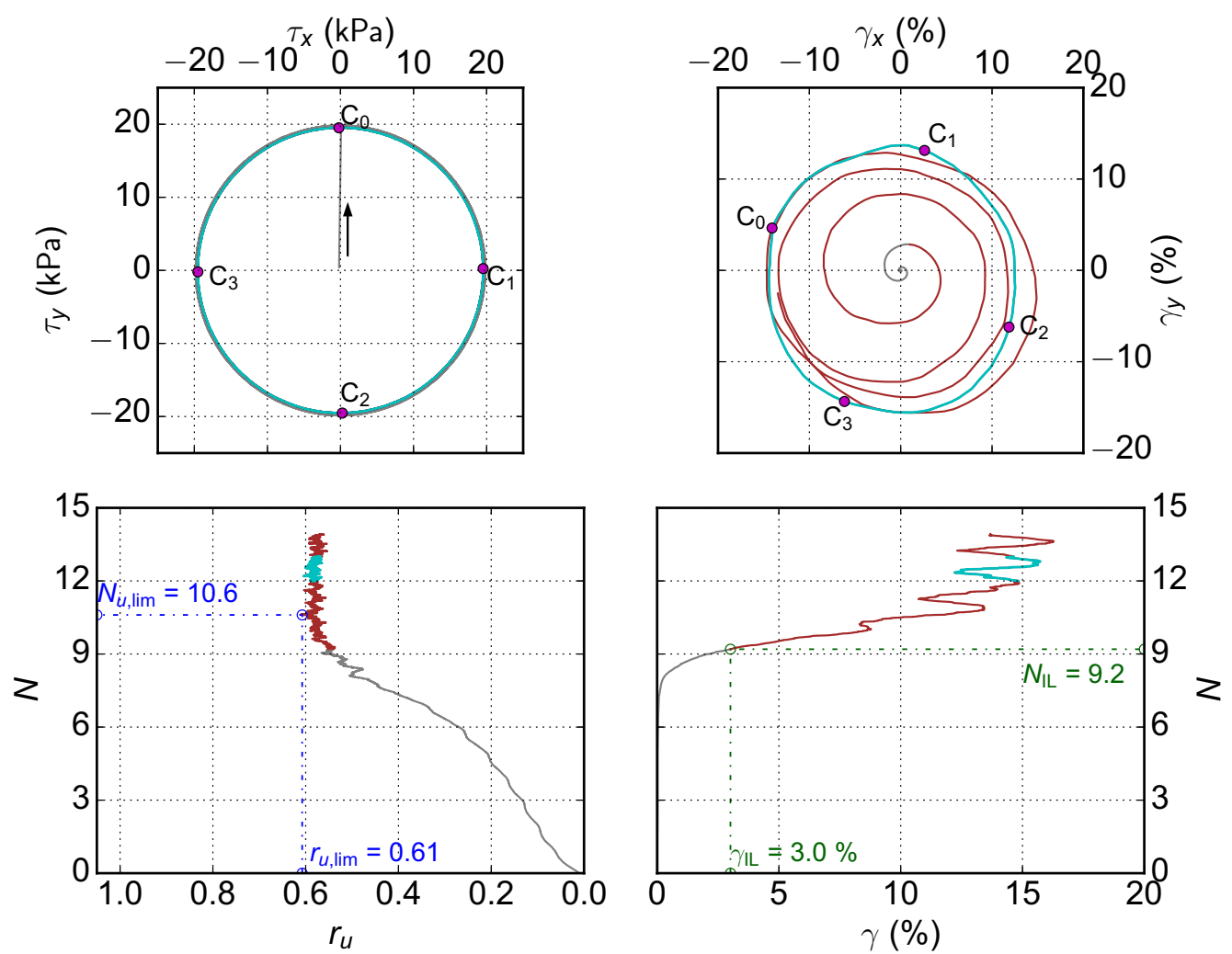

Fig. 7 Macroscopic response of constant-volume multidirectional cyclic shear test with $\mathrm{CSR}_{x}=\mathrm{CSR}_{y}=0.20$ : circular shear path 

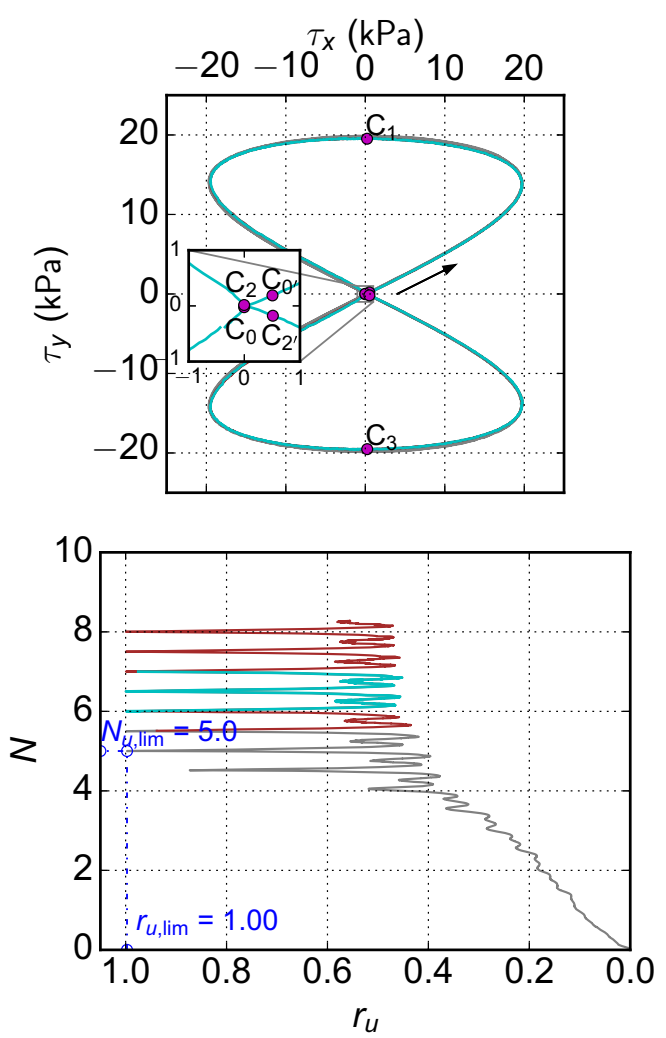
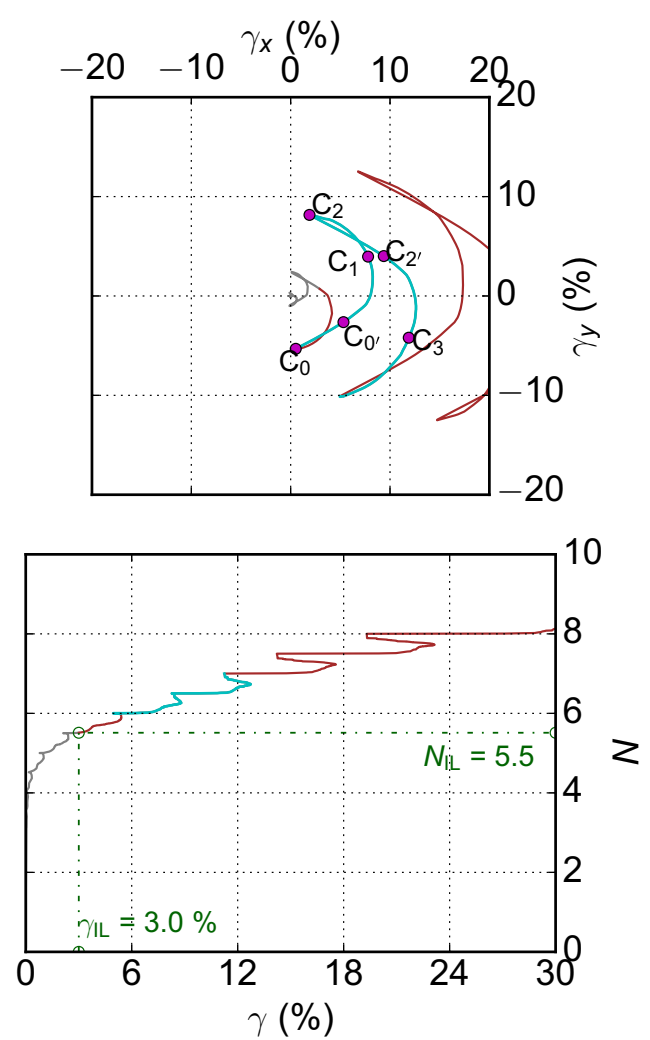

Fig. 8 Macroscopic response of constant-volume multidirectional cyclic shear test with $\mathrm{CSR}_{x}=\mathrm{CSR}_{y}=0.20$ : 8-like shear path

transiently, indicating the vanishing of $p$, whereas in 2-D linear and circular paths it remains well below 1.0. We also see that all shear paths generate large shear strains. These observations suggest that the vanishing of the mean effective stress is not a necessary condition for large shear strain development, although it holds for the cyclic simple shear test as observed in Fig. 5. Recalling the motivation behind the application of SSR concerning the stress state in sloping grounds, as SSR indicates the slope dip direction, Fig. 6 suggests that large shear strain develops mainly along the slope direction with the cyclic loading perpendicular to that direction. Moreover, the four simulations imply that $N_{\mathrm{IL}}$ and $N_{u, \text { lim }}$ are fairly close to each other, suggesting that initial liquefaction occurs almost simultaneously with $p$ dropping to its minimum. All these observations are consistent with the laboratory findings [6, 26, 28, 29].

A loading cycle in the post-liquefaction period of each simulation is selected to zoom into the details of the evolution of microscopic descriptors in Sect. 4. The related cycle is highlighted in cyan color in Figs. 5, 6, 7 and 8, along with some selected states. In particular, in the related loading cycle of 1-D linear and 8-like paths, six states $\mathrm{C}_{0}$, $\mathrm{C}_{0^{\prime}}, \mathrm{C}_{1}, \mathrm{C}_{2}, \mathrm{C}_{2^{\prime}}$, and $\mathrm{C}_{3}$, were selected with the prefix $\mathrm{C}$ representing cycle and the subscripts representing the sequence of occurrence of such states. $\mathrm{C}_{0}$ refers to the instance with the largest number of floaters (particles without any contact) when $\tau$ transitions from negative to positive (1-D linear) or from quadrant III to quadrant I in the space of applied shear stresses (8-like). $\mathrm{C}_{0^{\prime}}$ refers to when $r_{u} \leq 0.99$ for the first time after $\mathrm{C}_{0}$, the subsequent $\mathrm{C}_{1}$ refers to $\tau_{y}$ reaching the shear stress amplitude and $\mathrm{C}_{2}, \mathrm{C}_{2^{\prime}}$ and $\mathrm{C}_{3}$ are similar to $\mathrm{C}_{0}, \mathrm{C}_{0^{\prime}}$ and $\mathrm{C}_{3}$, respectively, but in the remaining loading cycle. $\mathrm{C}_{0^{\prime}}$ and $\mathrm{C}_{2^{\prime}}$ are missing in the shear paths of 2-D linear and circular, but the other particular states apply to these paths as well, and one can see those in in Figs. 6 and 7.

In particular, in the related loading cycle of 1-D linear and 8-like paths, six states were selected with the prefix $\mathrm{C}$ representing cycle: $\mathrm{C}_{0}$ refers to the instance with the largest number of floaters (particles without any contact) when $\tau$ transitions from negative to positive (1-D linear) or from quadrant III to quadrant $\mathrm{I}$ in the space of applied shear stresses (8-like). $\mathrm{C}_{0^{\prime}}$ refers to when $r_{u} \leq 0.99$ for the first time after $\mathrm{C}_{0}$, the subsequent $\mathrm{C}_{1}$ refers to $\tau_{y}$ reaching the shear stress amplitude and $\mathrm{C}_{2}, \mathrm{C}_{2^{\prime}}$ and $\mathrm{C}_{3}$ are similar to $\mathrm{C}_{0}$, $\mathrm{C}_{0^{\prime}}$ and $\mathrm{C}_{3}$, respectively, but in the remaining loading cycle. $\mathrm{C}_{0^{\prime}}$ and $\mathrm{C}_{2^{\prime}}$ are missing in the shear paths of 2-D linear and circular, but the other particular states apply to these paths as well, and one can see those in in Figs. 6 and 7. 


\subsection{Post-liquefaction pore pressure evolution}

The evolution of pore pressure ratio $r_{u}$ in Figs. 5, 6, 7 and 8 indicates that once the limit pore pressure ratio is achieved, the pore pressures oscillate [29]. Thus, for each simulated test, one can extract the limit pore pressure ratio $r_{u, \lim }$ and the corresponding shear stress ratio $\tau_{u, \lim } / p_{0}$, as shown in Table 2. Figure 9 shows $r_{u, \mathrm{lim}}$ versus $\tau_{u, \mathrm{lim}} / p_{0}$ for all simulations. Most of the data points can be fitted by a decreasing linear function $y=-2.08 x+1.00$, and the slope is very close to $-1 / M_{f}=-2.04$, where $M_{f} \simeq 0.49$ is the slope of stress path at butterfly shape as shown in Fig. 5. This value of $M_{f}$ is slightly higher but very close to the shear stress ratio $M_{c}=\tau / p$ at critical state of the constant-volume monotonic simple shear simulation while the latter is around 0.43 . Note that the fit function intercepts the $y$-axis at 1 , suggesting that $r_{u, \lim }=1$ occurs simultaneously with $\tau_{u \text { lim }}=0$. They are necessary and sufficient conditions for each other.

There are a few data points that do not fall on but are below the fitted line. They consist of three points from the 1-D linear path, three points from the 2-D linear path, and one point from the circular path. The three cases of 1-D linear path occur for $\mathrm{SSR}_{x}$ exceeding $\mathrm{CSR}_{y}$ so that there is no shear stress reversal, i.e., the minimum value of $\tau_{y}$ stays positive. For these tests, when $r_{u \text { lim }}$ is attained, the corresponding stress state $\left(\tau_{y}, p\right)$ is still away from the failure surface of Fig. 5, which is consistent with the laboratory experiments $[7,11,24,66,75]$. The three cases of 2-D linear path correspond to large values of $\mathrm{SSR}_{y}$. For the circular path, its stress path does not contain the origin (zero shear stress state) of the applied shear stress in the space $\left(\tau_{x}, \tau_{y}\right)$. Kammerer et al. [29] also pointed out certain similar cases where the imposed shear stress remained so large that the soil could not contract significantly upon loading reversal. Hence, the decrease in mean effective

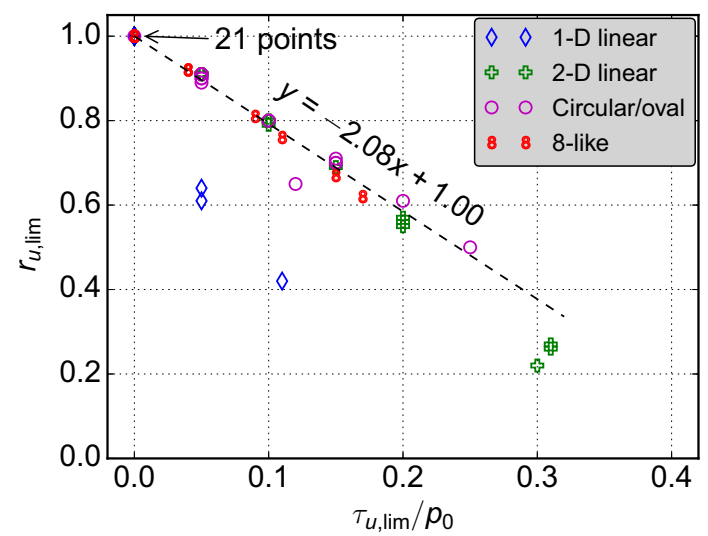

Fig. 9 Relationship between the limit pore pressure ratio $r_{u, \lim }$ and the corresponding shear stress ratio $\tau_{u, \lim } / p_{0}$ stress is not large enough to bring the granular system close to the Coulomb failure surface.

\subsection{Cyclic liquefaction resistance}

Cyclic liquefaction failure is often defined as the state at which shear strain exceeds a limit value, taken to be $3 \%$ in the present work. Cyclic liquefaction can be triggered by different combinations of uniform CSR, and the number of loading cycles. The liquefaction strength curve, i.e., the plot of CSR versus the number of cycles to the initial liquefaction $N_{\mathrm{IL}}$, is of great practical importance.

In developing the CSR $-N_{\mathrm{IL}}$ curves, the CSR must be defined differently for different test types. The cyclic shearing stages of the 1-D and 2-D linear shear paths require only $\mathrm{CSR}_{y}$, while the circular/oval and 8-like shear paths require both $\operatorname{CSR}_{x}$ and $\operatorname{CSR}_{y}$. To facilitate the comparative analysis of the liquefaction strength curve, we select $\mathrm{CSR}=\mathrm{CSR}_{y}$ as the control variable, and for circular and 8-like shear paths we only consider the cases with $\mathrm{CSR}_{x}=\mathrm{CSR}_{y}$, i.e., aspect ratio (AR) of 1 . Recall that Figs. 5, 6, 7 and 8 are simulations with $\mathrm{CSR}_{y}=0.20$, so that additional simulations with different levels of $\mathrm{CSR}_{y}$ are needed to analyze the cyclic liquefaction strength. For generating the CSR $-N_{\mathrm{IL}}$ curves, the selected 1-D linear, circular, and 8-like shear paths were those with zero SSR. For the 2-D linear case, however, $\mathrm{SSR}_{x}=0.10$.

Figure 10 presents the liquefaction strength curves for the four types of shear paths where the initial liquefaction is defined as the first time the total shear strain $\gamma=$ $\left(\gamma_{x}^{2}+\gamma_{y}^{2}\right)^{1 / 2}$ reaches $3 \%$. For each shear path, three data points are shown. We see that the liquefaction resistance declines with increasing $\mathrm{CSR}_{y}$, as expected. Following the convention in soil dynamics, the liquefaction strength data points can be fitted by a power-law function [25, e.g.,]:

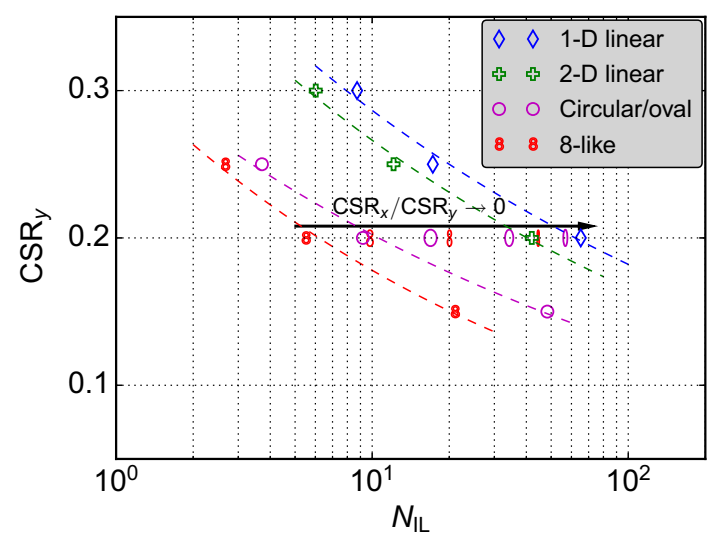

Fig. 10 Cyclic liquefaction strength curves for different shear stress paths. The dashed lines are power-law fits to the data points 
$\mathrm{CSR}_{y} \propto N_{\mathrm{IL}}^{-b}$

where the exponent is found to be $b \simeq 0.20$ for the four paths. We also observe a decrease in liquefaction resistance following the order of 1-D linear, 2-D linear, circular, and 8-like shear paths, in agreement with laboratory findings [28]. This decrease in $N_{I L}$ for two-dimensional cyclic paths can be attributed to the shear stress dip in 2-D linear path and stress rotation in circular and 8-like paths in Fig. 10, which lead to extra degradation of the granular load-bearing structure and therefore lower level of liquefaction resistance.

To explore the effect of AR on the cyclic liquefaction resistance, four additional simulations of circular/oval and 8-like shear paths with different values of $\mathrm{CSR}_{x}$ and a fixed value of $\mathrm{CSR}_{y}=0.20$ are added to Fig. 10; the data points are shown with thinner oval and 8-like symbols. The collective data points from the tests at $\mathrm{CSR}_{y}=0.20$ indicate an increase in liquefaction resistance with a decreasing $\mathrm{AR}$ as they approach the 1-D linear case, in agreement with experiments $[26,28]$. They coincide with the 1-D linear case as AR tends to zero.

To explore the effect of static shear stress on cyclic liquefaction resistance, Fig. 10 can be extended by incorporating liquefaction strength curves for different values of $\mathrm{SSR}_{[]}$. Here the bracket subscript [] represents $y$ for 1-D linear and circular shear paths and $x$ for 2-D linear and 8like shear paths, as illustrated in Fig. 1. In doing so, one obtains many fitted curves to the corresponding simulation data using Eq. 10. The simulation data cover different shearing paths and different values of $\operatorname{SSR}_{[]}$. To characterize the cyclic resistance ratio (CRR) of the sample in a certain shearing path and under a certain value of $\operatorname{SSR}_{[1}$, the value of $\mathrm{CSR}_{y}$ required to cause initial liquefaction at 10 loading cycles, denoted hereafter as $\mathrm{CRR}_{y}$, can be extracted from the fitted curves. Element level experimental studies on some of these loading paths on sands were reported in $[6,24,65,76]$. For developing the $\mathrm{CRR}_{y}-$ $\mathrm{SSR}_{[]}$plot, and particularly for the circular and 8-like paths, we only use the simulations with $\mathrm{AR}=1$, hence excluding the effect of AR. Therefore, in total, we use 51 out of the 57 simulations presented in Table 2. Figure 11 shows the values of $\mathrm{CRR}_{y}$ against $\mathrm{SSR}_{[]}$for the four shear paths. The dashed line shows the line $\mathrm{CRR}_{y}=\mathrm{SSR}_{[]}$ dividing the parameter space into two regions. Depending on the stress path, the points above and below this line could have different meanings. For the 1-D linear and circular shear paths, being above this line means experiencing the change of $\tau_{y}$ sign in each loading cycle for the corresponding cyclic stress ratio, and vice versa for below the line. The same argument is valid for the 8-like shear path, but only in terms of the change of $\tau_{x}$ sign in each

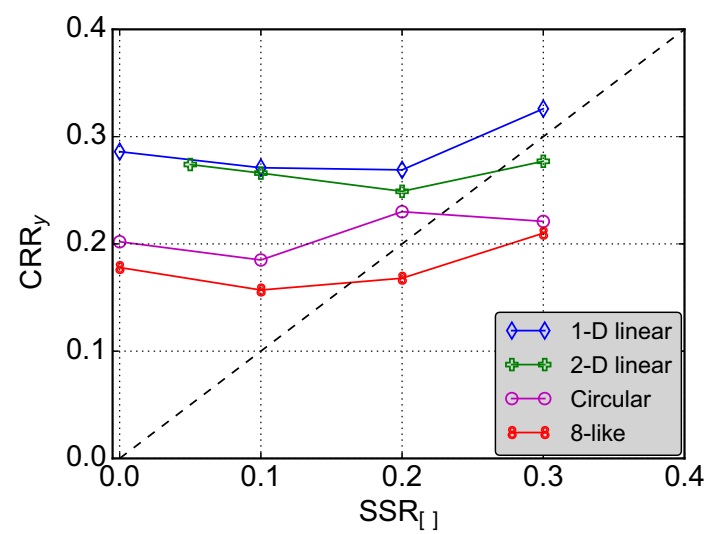

Fig. 11 Cyclic liquefaction resistance as a function of the level of static stress ratio

loading cycle. These arguments do not apply to the 2-D linear shear path.

A marked feature in Fig. 11 is that $\mathrm{SSR}_{[]}$affects the cyclic resistance differently for these shear paths. For the 1-D linear shear path, $\mathrm{CRR}_{y}$ presents an increasing trend with increasing $\mathrm{SSR}_{y}$, and for the examined level of $\mathrm{SSR}_{[]}$ in this study, the curve stays above the dashed line, i.e., in the region with shear stress reversal. This is similar to the observations from laboratory experiments $[24,76]$. The 2D linear shear path curve stays below the 1-D linear one, and it does not change significantly, consistent with the laboratory results on medium dense sands [6]. The curves of circular and 8-like shear paths are located even below the 2-D linear one, and both cross the line $\mathrm{CRR}_{y}=\mathrm{SSR}_{[]}$. We could not find any corresponding laboratory study in the literature revealing the effect of static shear stress on cyclic liquefaction resistance for circular and 8-like shear paths in such a systematic way. While Fig. 10 clearly shows the effect of shear path on the cyclic resistance curves in absence of $\mathrm{SSR}_{[]}$, Fig. 11 shows that $\mathrm{SSR}_{[]}$can have an obvious impact when SSR is large. For example, in this figure, around $\mathrm{SSR}_{x}=0.20$ the $\mathrm{CRR}_{y}$ values of $2-\mathrm{D}$ linear and circular shear paths are similar. The same thing can be seen around $\mathrm{SSR}_{x}=0.30$ for the circular and 8-like shear paths.

Kammerer et al. [29] highlighted two conflicting effects of the imposed shear stress. As previously shown, for the stress paths where $\min \left(\tau_{x}^{2}+\tau_{y}^{2}\right)^{1 / 2} / p_{0}$ approaches 0 transiently, $r_{u, \text { lim }}$ approaches 1 , where large shear strain can develop due to the vanishing resistance of the granular system. For the stress paths where $\min \left(\tau_{x}^{2}+\tau_{y}^{2}\right)^{1 / 2} / p_{0}$ is above $0, r_{u, \text { lim }}$ stays below 1 ; however, the imposed shear stress can still drive the system to deform progressively. For 1-D linear shearing, the former scenario corresponds to occurrence of shear stress reversal and large cyclic strains due to the state $r_{u} \simeq 1$, and the latter scenario corresponds 
to no shear stress reversal but development of residual deformation, as also noted in [11, 24]. More specifically, the main origin of reaching the $\gamma_{\mathrm{IL}}=3 \%$ in the 1 -D linear cases of Fig. 10 is the first effect, whereas in the 2-D linear and circular cases the large strains arise as a result of the second effect. For the 8-like shear paths, both of these effects are at work to increase the shear strain beyond $\gamma_{\mathrm{IL}}=3 \%$. This can be observed by comparing the strain path in Fig. 8 from $\mathrm{C}_{0}$ to $\mathrm{C}_{0^{\prime}}$ (where the first effect is dominant) with the one from $\mathrm{C}_{0^{\prime}}$ to $\mathrm{C}_{1}$ (where the second effect is dominant). Given these two effects at low and high static shear stresses, naturally one would expect that at an intermediate level of static shear stress CRR attains a minimal level. Figure 11 indicates that the lowest value of $\mathrm{CRR}_{y}$ is reached along with a certain amount of static shear stress, quite in line with the suggestion that the balance between these two competing aspects results in high shear strain potential [29].

\section{Granular microstructure}

This section first analyzes the particle connectivity and friction mobilization, which are correlated with system stability. Then, a particle-void fabric analysis is adopted to clarify the mechanisms underlying the macro deformation. Finally, the fabric and force anisotropies are used to partition the load-bearing network, revealing the particle-scale origins of shear resistance under cyclic loading and in the transition to the cyclic liquefaction state.

\subsection{Particle connectivity and friction mobilization}

The coordination number $z_{\mathrm{g}}$ is the average number of force-bearing contacts per particle excluding floaters (particles without contacts):

$z_{\mathrm{g}}=\frac{2 N_{c}}{N_{p}-N_{p}^{0}}$,

where $N_{p}$ is the total number of particles and $N_{p}^{0}$ is the number of floaters. In addition to $z_{\mathrm{g}}$, also named "geometrical coordination number" [64], one may also calculate the average coordination number considering all the particles and the "mechanical coordination number" considering particles with at least two contacts [63]. Here $z_{\mathrm{g}}$ is used instead of the other two quantities because it better approximates the level of static redundancy in the system, i.e., the difference between the total number of constraints and the total number of degrees of freedom. Thus, it is more closely linked with the granular system's overall stability since a minimum number of contacts are necessary for the static equilibrium of all particles. Its decrease below this minimum value reflects the system's destabilization. Figure 12 shows the evolution of $z_{\mathrm{g}}$ along with the cyclic shearing process for the four simulations of Figs. 5, 6, 7, and 8 . The time histories are colored according to the value of $r_{u}$ ranging between 0 and 1. A zoomed-up window for each test is added to show the variation of $z_{\mathrm{g}}$ in a loading cycle in the post-liquefaction period, and the highlighted dots represent selected states. We observe an overall gradual decreasing trend of $z_{\mathrm{g}}$ from 4.7 down to a value below 4 along the cyclic shearing for all the four shear paths. After the state of $\min \left(N_{u, \text { lim }}, N_{\mathrm{IL}}\right)$, for 1-D linear and 8-like shear paths, $z_{\mathrm{g}}$ tends to oscillate significantly and drops below 3.6 when $r_{u}$ exceeds 0.99 ; for the 2-D linear and circular shear paths, $z_{\mathrm{g}}$ oscillates mildly and stays above 3.6.

As mentioned previously, $z_{\mathrm{g}}$ can also be viewed as an approximate measurement of the level of static redundancy in the system, i.e., the total number of constraints compared to the total number of degrees of freedom. For the 3DDEM with contact laws consisting of the normal collision, tangential sliding, rolling, and torsion, each contact involves three forces and three torques (constraints) to be determined. On the other hand, the number of equations equals six force/torque balance equations for the degrees of freedom of each particle. Hence, removing the floating particles and equating the number of degrees of freedom with the number of forces/torques, we come up with the relation $6 N_{c}=6\left(N_{p}-N_{p}^{0}\right)$ for an isostatic system with infinite friction. Thus, the critical value of $z_{\mathrm{g}}$ for isostaticity is $z_{\text {iso }}=2$. For a system with finite friction, the value of $z_{\text {iso }}$ may slightly increase due to the mobilization of sliding, rolling, or torsion friction, thus reducing the number of constraints. For a system with sliding but zero rolling and torsion, we have $z_{\text {iso }}=6 /\left(3-f_{\mathrm{s}}\right)$ where $f_{\mathrm{s}}$ is the sliding mobilization fraction; for a system with sliding but infinite rolling and torsion, we have $z_{\text {iso }}=12 /\left(3-f_{\mathrm{s}}\right)$ [22]. However, one cannot express $z_{\text {iso }}$ theoretically since $z_{\mathrm{g}}$ cannot be determined only by the combinations of each friction mobilization fraction. Instead, another descriptor called "redundancy index" is introduced and may provide a better choice $[18,23,31,38,49,81]$. Still one can treat $z_{\text {iso }}=2$ as the lowest bound for an under-constrained system. As to the upper bound, given the small values of rolling and torsion friction in this study, the value $z_{\text {iso }} \leq 3$ should be a reasonable guess. According to Fig. 12a, one can assert that along the 1-D linear shear path, the system becomes transiently under-constrained $\left(z_{\mathrm{g}}<2\right)$. For the $2-\mathrm{D}$ linear and circular shear paths, the system remains overconstrained $\left(z_{\mathrm{g}}>2\right)$ as $z_{\mathrm{g}}$ is always well above 3 . In the 8like shear path, the system may become under-constrained 


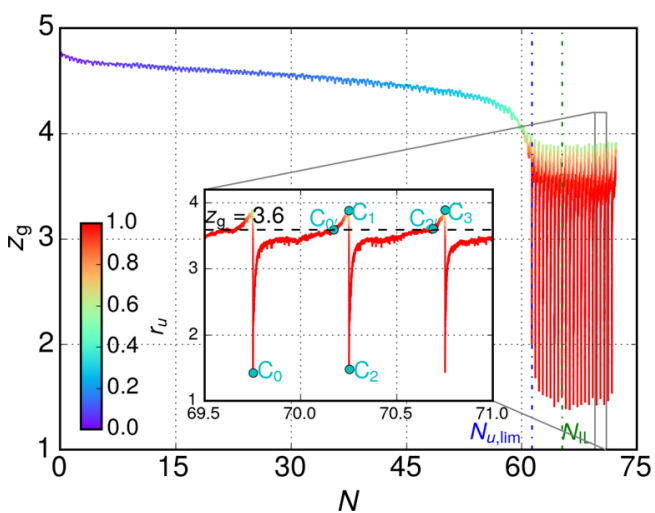

(a)

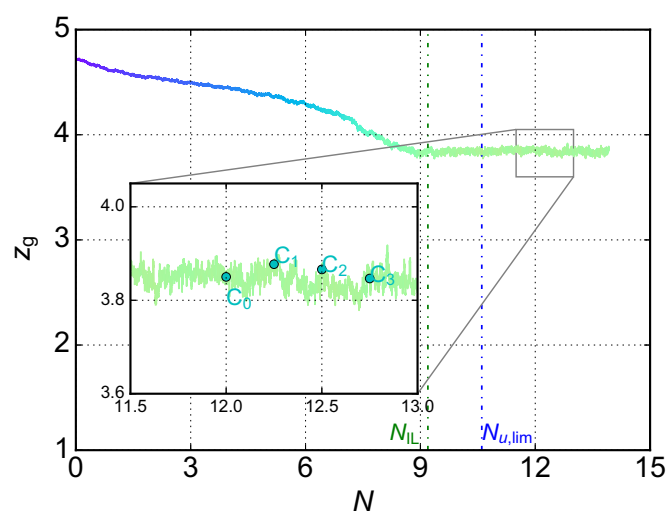

(c)

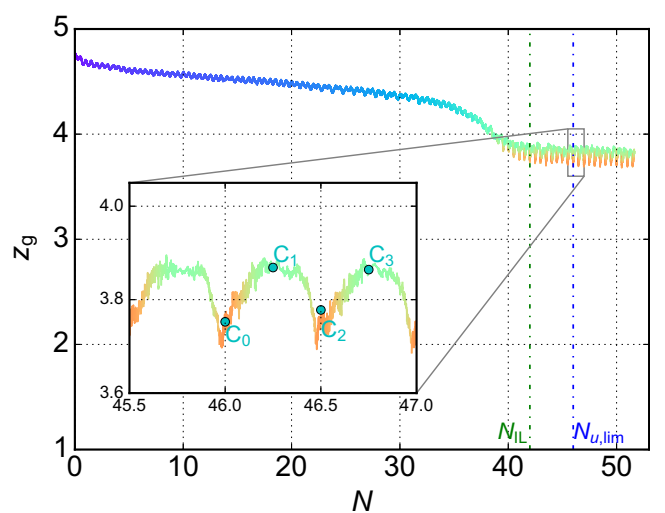

(b)

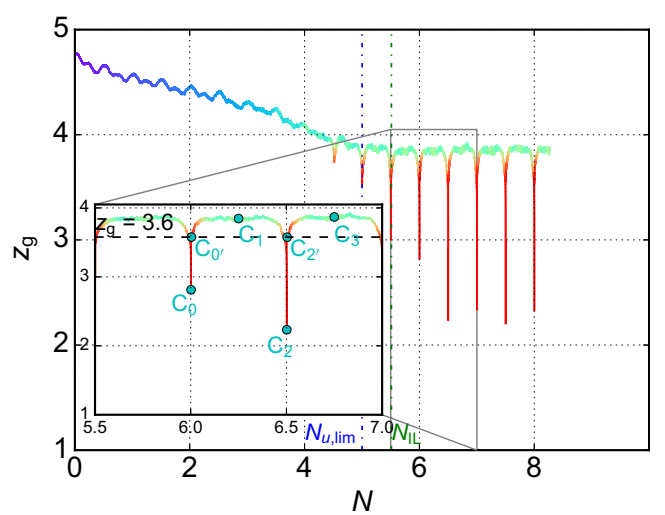

(d)

Fig. 12 Evolution of the coordination number $z_{\mathrm{g}}$ under different shear paths: a 1-D linear; b 2-D linear; c circular; d 8-like

temporally given that the recorded minimum value of $z_{\mathrm{g}}$ is quite close to 2 .

Let us go back to the particular states highlighted in Figs. 5, 6, 7, and 8, as well as the two reference states IP and CS. Figure 13 presents the corresponding connectivity $P(c)$, defined as the proportion of particles with exactly $c$ contacts. Compared with IP, the monotonic and cyclic loading paths push the distribution leftwards (smaller values of $c$ ) in two different ways. The monotonic loading mainly changes the distribution for $c \leq 4$ while cyclic loading drags the whole distribution leftwards. In 1-D linear and 8-like shear paths, an irregular distribution is observed with a higher proportion of floaters at $\mathrm{C}_{0}$ and $\mathrm{C}_{2}$. These are the states corresponding to the lowest $z_{\mathrm{g}}$, revealing the collapse of the contact network. For the other selected states, the contact network is well formed. We observe also a remarkable change of $P(c)$ between $\mathrm{C}_{0}$ and $\mathrm{C}_{0^{\prime}}$, or $\mathrm{C}_{2}$ and $\mathrm{C}_{2^{\prime}}$, despite the proximity of the mean stresses. As long as $r_{u} \geq 0.99$, the sample deforms significantly to rebuild the contact network without a significant change of $p$. Hence, this process is more akin to a redistribution of forces among particles rather than a buildup of stress across the sample. For 2-D linear and circular paths, consistently with the system's over-constrained state, the distribution does not vary noticeably.

A local coordinate system $(\boldsymbol{n}, \boldsymbol{t})$ can be attached to each contact, with $\boldsymbol{n}$ being the unit vector perpendicular to the contact plane and $\boldsymbol{t}$ an orthonormal unit vector in the contact plane oriented along the tangential contact force. Thus, we have $\boldsymbol{f}=f_{n} \boldsymbol{n}+f_{t} \boldsymbol{t}$, with $f_{n}$ and $f_{t}$ representing the magnitudes of the normal and tangential forces, respectively. To quantify the susceptibility of a contact to sliding, which is associated with soil plasticity [2], one can define the friction mobilization index [3, 37]:

$I_{m}=\left|f_{t}\right| /\left(\mu_{t} f_{n}\right)$

where $\mu_{t}$ is the friction coefficient. This index varies between 0 and 1 , with 1 indicating sliding or fully mobilized contact.

Figure 14 displays the snapshots of the probability density function $P\left(I_{m}\right)$ of $I_{m}$ at selected states for the four shear paths where IP and CS are added for comparison. Compared with IP, both monotonic and cyclic loading tend to push $P\left(I_{m}\right)$ rightwards, i.e., a larger proportion of contacts getting close to sliding. Interestingly, the sliding contact proportion $f_{\mathrm{s}}$ at CS is quite similar to the value at 


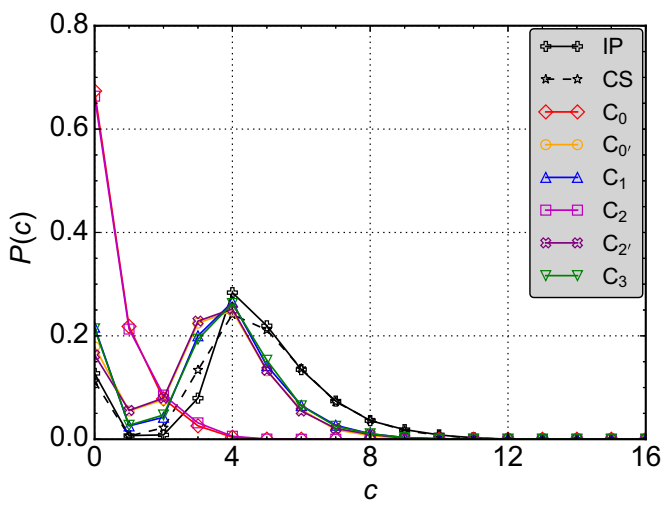

(a)

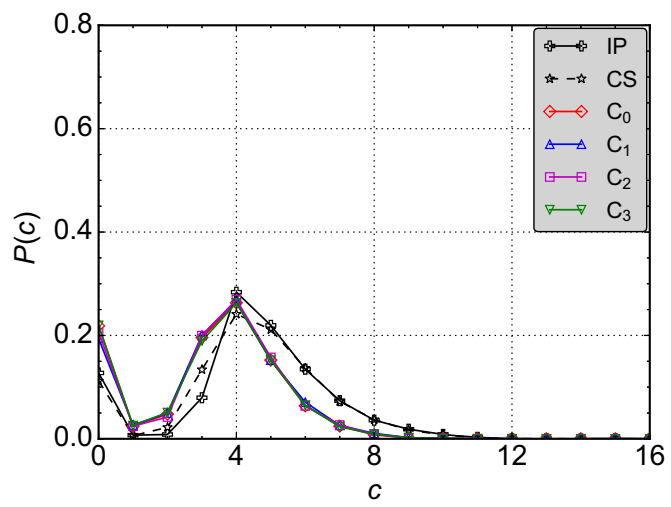

(c)

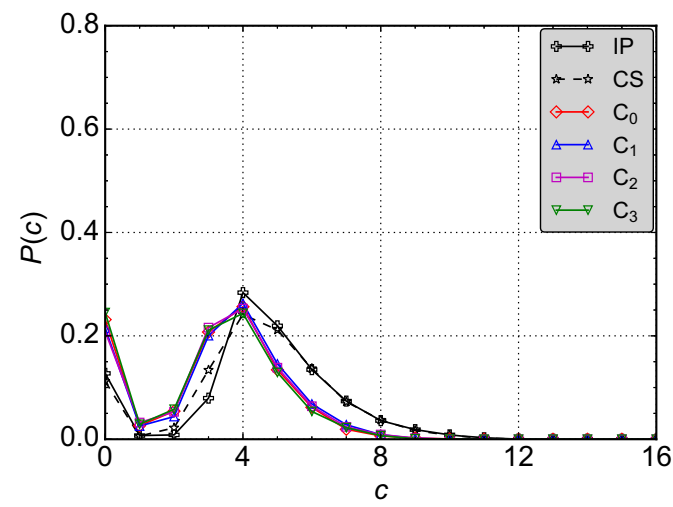

(b)

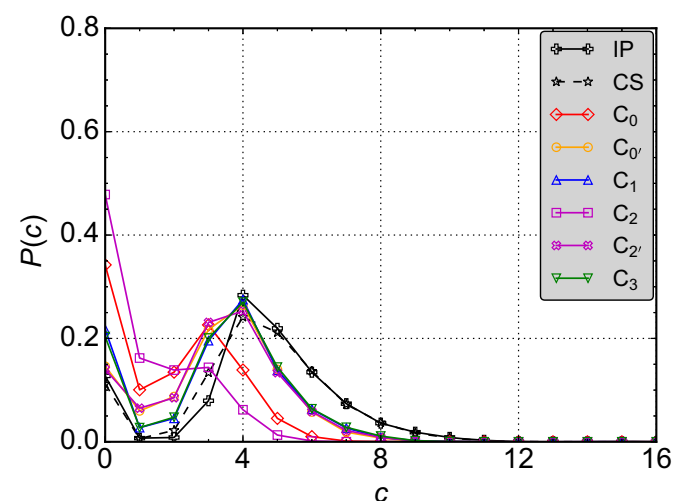

(d)

Fig. 13 Connectivity diagram expressing the fraction $P(c)$ of particles with exactly $c$ contacts at particular states in shear path of: a 1-D linear; b 2-D linear; c circular; d 8-like

IP, around $0.2 \%$. For cyclic loading, the sliding proportion varies significantly depending on the selected states. At $\mathrm{C}_{0}$ and $\mathrm{C}_{2}$, where the shear stress vanishes for 1-D linear and 8-like shear paths, one can observe a large proportion of fully mobilized contacts as shown in the inset windows. The corresponding sliding contact proportions for the 1-D linear and 8-like shear paths are around $60 \%$ and $10 \%$, respectively. The difference between 1-D linear and 8-like plots is only due to time resolution, i.e., the recording frequency of data. The proportion of mobilized contacts decreases with increasing shear stress [19], as verified at the states $\mathrm{C}_{1}$ and $\mathrm{C}_{3}$ where $f_{\mathrm{s}}$ is around $2 \%$ for all four cyclic stress paths. By comparing $\mathrm{C}_{0}$ with $\mathrm{C}_{0^{\prime}}$ or $\mathrm{C}_{2}$ with $\mathrm{C}_{2^{\prime}}$ where $f_{\mathrm{s}}$ at $\mathrm{C}_{0^{\prime}}$ or $\mathrm{C}_{2^{\prime}}$ is around $10 \%$, one can conclude that the force network is still not fully established despite the resilience of the contact network in Fig. 12a and d. Given the stability of the system for 2-D linear and circular shear paths, it is expected that $P\left(I_{m}\right)$ does not vary significantly, as observed in Fig. 14b and c although Fig. 14b exhibits a slight increase in the number of mobilized contacts at $\tau$ reaching its minimum value $\left(\mathrm{C}_{0}\right.$ or $\left.\mathrm{C}_{2}\right)$. It should also be remarked that the distribution of $I_{m}$ has a peak value around $I_{m} \simeq 0.3$ independently of the shear path. This feature reflects the anisotropy of the contact network with friction mobilization depending on the orientations of the contacts.

Figure 15 shows snapshots of normal forces at $\mathrm{C}_{2}$ for each shear path. This is the system's weakest state in a post-liquefaction loading cycle for all paths but the circular one. The forces are represented as bars joining particle centers with bar thickness proportional to the intensity of the normal force. The bar is colored according to the value of $I_{m}$ at each contact. In addition, Fig. 16b presents a zoom-in view of Fig. 15a to show the details, where the color code is replaced by the value of $f_{n}$, with Fig. 16a at IP for the comparison. In this "unjammed" state, we observe the scattering of weak and short force chains with a large proportion of mobilized contacts for 1-D linear and 8-like shear paths $[4,21]$, and a span of strong and long force chains connecting the top and bottom walls for 2-D linear and circular shear paths, again compatible with quasistatic equilibrium. 


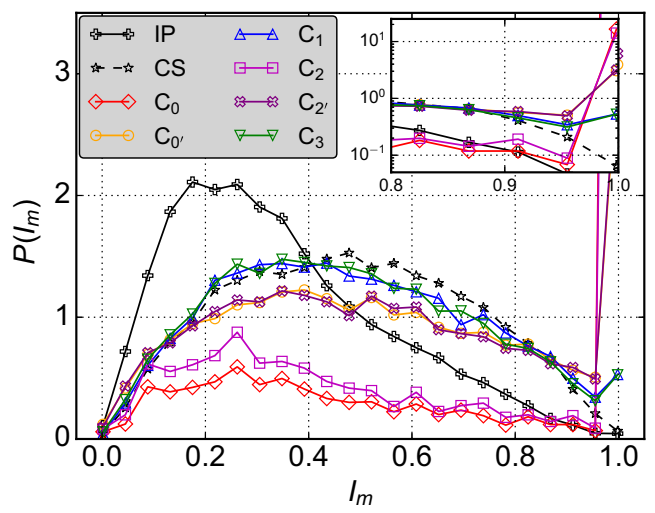

(a)

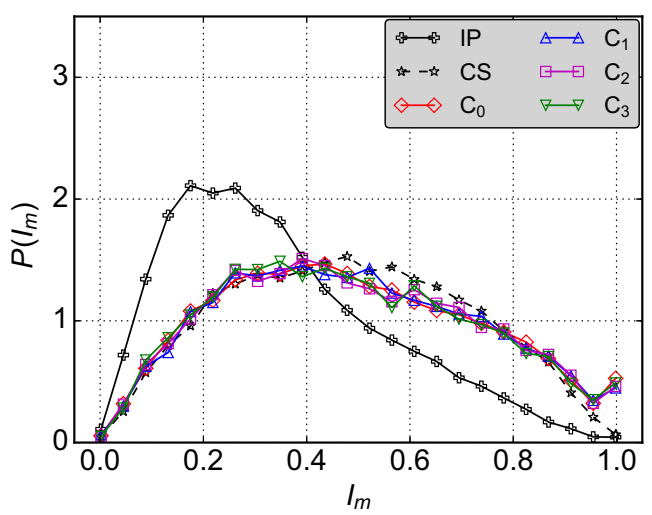

(c)

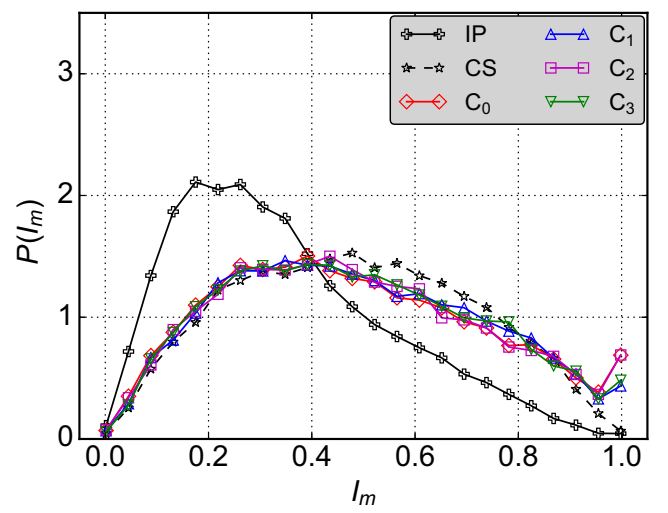

(b)

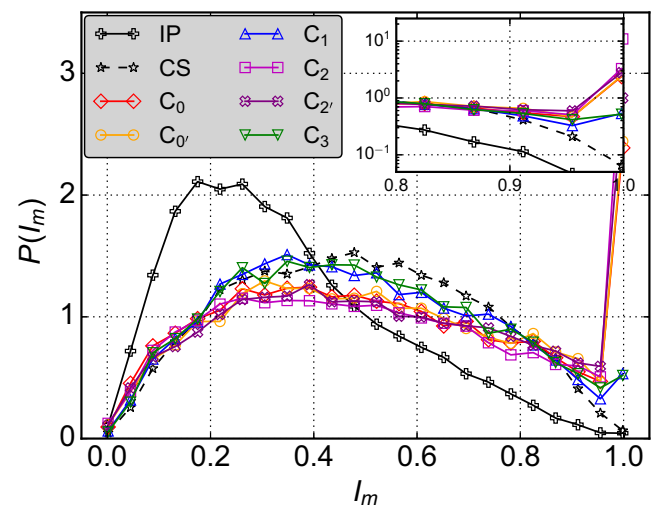

(d)

Fig. 14 Probability density function of friction mobilization index at selected particular states for different shear paths: a 1-D linear; b 2-D linear; c circular; d 8-like

\subsection{Particle-void fabric}

We have seen that the system falls into an under-constrained state occasionally for 1-D linear and 8-like shear paths and remains over-constrained for 2-D linear and circular paths. A related natural question is why large shear strain still develops in the 2-D linear and circular tests, as observed in Figs. 6 and 7. The analysis of stress fluctuations during monotonic loading [33] may shed light on this issue as local failure probably due to a multi-slip mechanism occurs very frequently despite the overall stability of the system. This local failure reduces the shear resistance gradually, inducing a mild increase in shear strain. In drained unidirectional cyclic loading, an abrupt reduction in the number of sliding contacts is observed upon transition from loading to reverse loading [1], leading to higher resistance. The distinct resistance between loading and reverse loading causes the accumulation of shear strain along one direction, i.e., the mode of development of residual deformation (cyclic ratcheting). We may also resort to void-related fabrics to search for some hints given that the voids are directly related to the strain [32].
Let us consider a particle-void indicator called "centroid distance" $D_{c}$ introduced by $[68,73]$. For each particle $i$, one can obtain its circumscribed Voronoi cell, and thus the vector connecting the cell center $\boldsymbol{O}^{i}$ and particle center $\boldsymbol{P}^{i}$, also normalized by the mean radii of particles $R_{50}$ :

$\boldsymbol{D}_{c}^{i}=\frac{\boldsymbol{P}^{i}-\boldsymbol{O}^{i}}{R_{50}}$

As a particle $i$ surrounded by a large Voronoi cell corresponds to a large value of $\left\|\boldsymbol{D}_{c}^{i}\right\|$, the latter can be used to quantify the distribution of voids surrounding the particles [73]. The centroid distance $D_{c}$ is the average value of $\left\|\boldsymbol{D}_{c}^{i}\right\|$ over all the particles in the system:

$D_{c}=\frac{1}{N_{p}} \sum_{i \in N_{p}}\left\|\boldsymbol{D}_{c}^{i}\right\|$.

Figure 17 displays the evolutions of $D_{c}$ for the four shear paths, along with the inset window for zooming in the selected loading cycle. The time history in each subplot is colored by the developed shear strain, as shown in the colorbar. Generally, for the four shear paths, at the beginning of cyclic loading before initial liquefaction, $D_{c}$ 


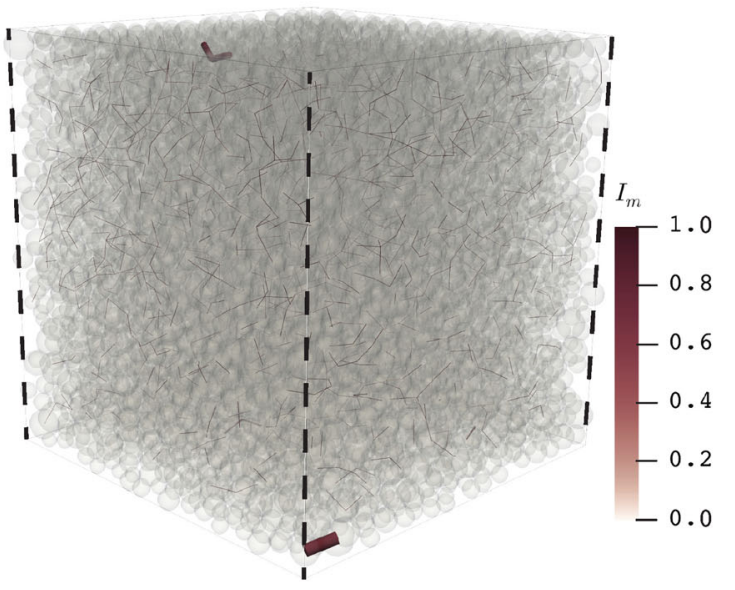

(a)

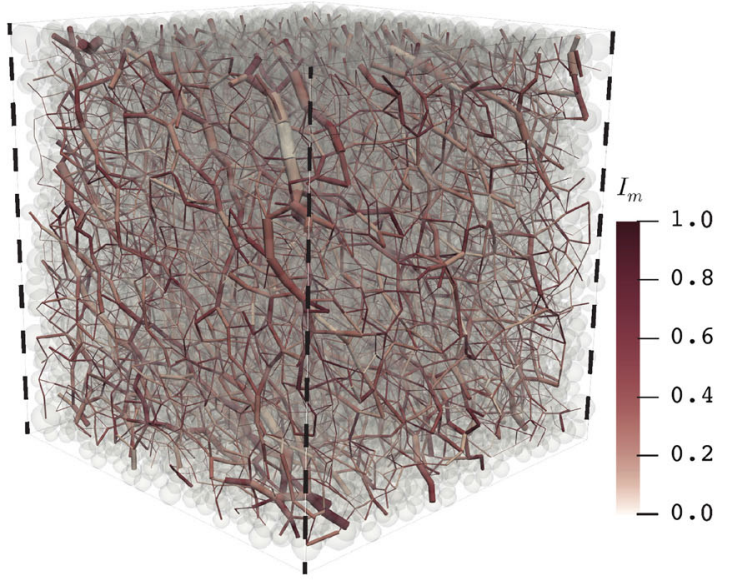

(c)

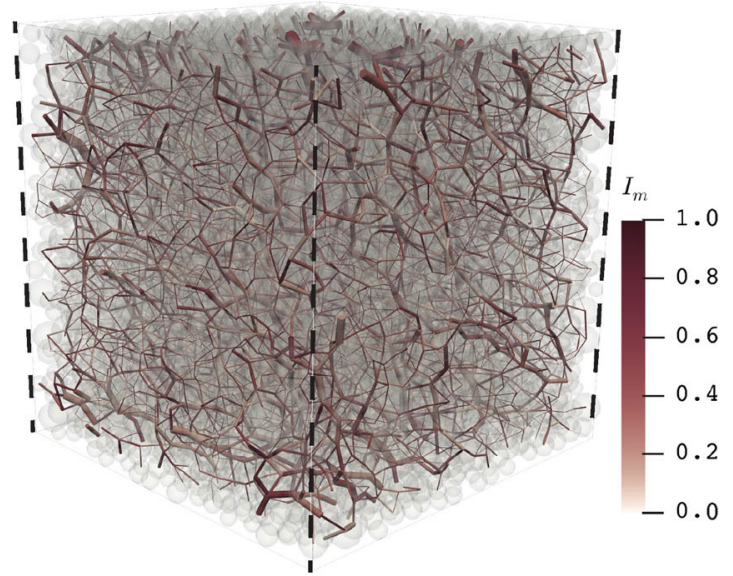

(b)

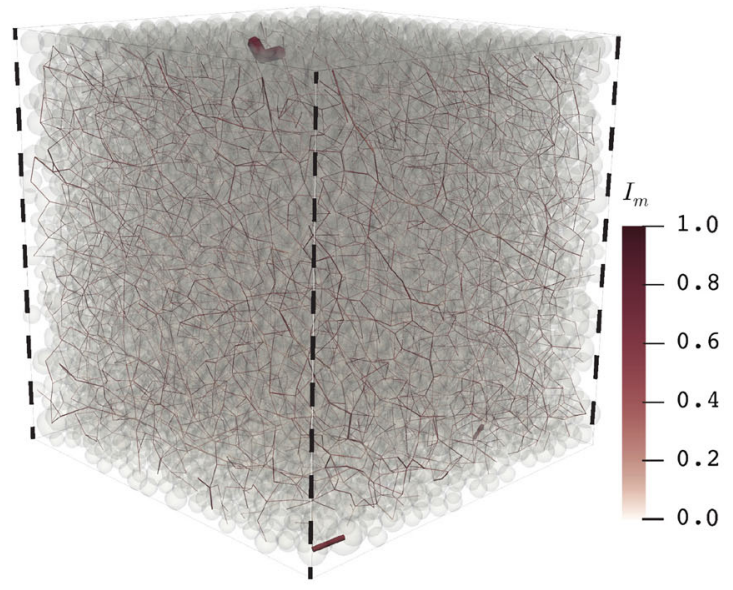

(d)

Fig. 15 Snapshot of normal forces in the sheared sample at $\mathrm{C}_{2}$ for different shear paths: a 1-D linear; b 2-D linear; c circular; $\mathbf{d}$ 8-like. Line thickness is proportional to the normal force at each contact. Color code represents the mobilized friction index $I_{m}$ in the range between 0 and 1 . The same camera view as Fig. 2 is used here (color figure online)

does not change noticeably, then drops significantly near initial liquefaction, and follows a decreasing trend during the post-liquefaction period. The values of $D_{c}$ at the initial liquefaction are very close (around 0.0704) for 1-D linear, circular, and 8-like paths, and smaller than that of 2-D linear (around 0.0720), which may indicate that there still exist large voids in the test of 2-D linear compared with the others. This may be because in the 2-D linear test, the packing network is not fully destroyed according to Fig. 12b, thus constraining the redistribution of voids due to cyclic shearing. One can notice the coincidence of a significant drop of $D_{c}$ and large shear strain accumulation, illustrating the direct link between void redistribution and large shear strain development. From that perspective, the pre-liquefaction period can be viewed as the stage with gradual propagation of cyclic disturbance to the whole sample, generating more connected but smaller voids. The
2-D linear and circular tests show a trend for saturation of $D_{c}$ in the last few loading cycles. However, it is not reflected by the 1-D linear and 8-like paths possibly because more loading cycles are needed. In the zoom-in windows of 1-D linear and 8-like tests, significant change mainly occurs between $\mathrm{C}_{0}$ and $\mathrm{C}_{0^{\prime}}$, or $\mathrm{C}_{2}$ and $\mathrm{C}_{2^{\prime}}$, corresponding to the deformation mode of cyclic mobility. In the zoom-in windows of 2-D linear and circular tests, the change tends to happen randomly in the loading cycle, a behavior that may be linked to the mode of residual deformation development. Interestingly, in the 1-D linear zoomed-in view, $D_{c}$ attains the local minima at nearly zero shear strain, as indicated by the color code. 


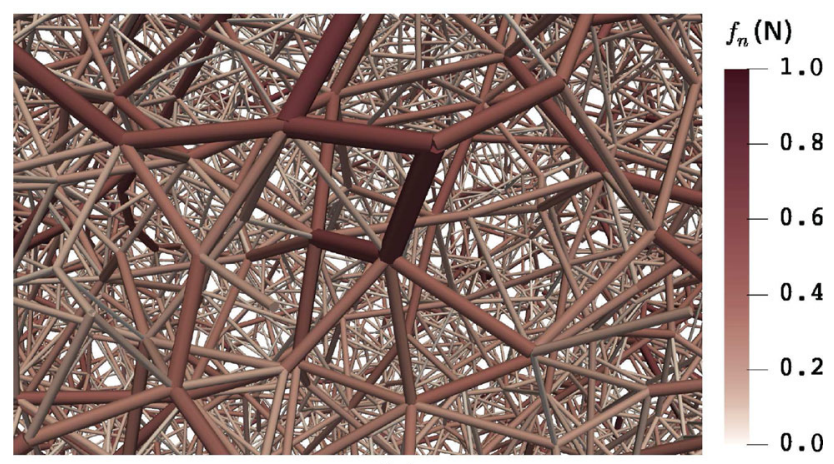

(a)

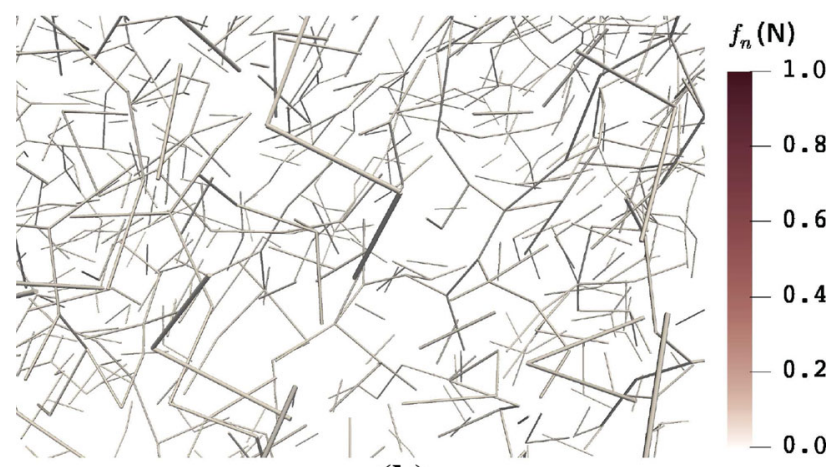

(b)

Fig. 16 Contact force networks for the system at $\mathbf{a}$ IP and $\mathbf{b} \mathrm{C}_{2}$ of 1-D linear path. Line thickness and color are proportional to normal contact force (color figure online)

\subsection{Fabric and force anisotropies}

The scalar variables considered so far do not account for the vectorial nature of the force network and strain-induced anisotropy, which play a crucial role in the mechanics of granular materials $[52,55]$. The geometrical anisotropy in a granular system can be described by the fabric tensor $\phi_{c}$, which describes the distribution of contact normals $\boldsymbol{n}$ $[46,56]$. It is defined as

$\boldsymbol{\phi}_{c}=\frac{1}{N_{c}} \sum_{c \in N_{c}} \boldsymbol{n} \otimes \boldsymbol{n}$,

where $\boldsymbol{n}$ is unit vector along the contact normal and $N_{c}$ is the total number of contacts. The fabric anisotropy tensor is defined by

$\boldsymbol{a}_{c}=\frac{15}{2}\left(\boldsymbol{\phi}_{c}-\frac{1}{3} \boldsymbol{I}\right)$,

where $\boldsymbol{I}$ is the second-order identity tensor. One can refer to [30] for the details of derivation.

In the same way, the contact force anisotropy can be extracted from force tensors that reflect the distribution of normal and tangential contact forces [30, 48, 59]. They can be considered as force-weighted fabric tensors and defined as

$$
\begin{aligned}
\boldsymbol{\phi}_{n} & =\frac{1}{N_{c}} \sum_{c \in N_{c}} \frac{f_{n} \boldsymbol{n} \otimes \boldsymbol{n}}{1+\boldsymbol{a}_{c}:(\boldsymbol{n} \otimes \boldsymbol{n})} \\
\boldsymbol{\phi}_{t} & =\frac{1}{N_{c}} \sum_{c \in N_{c}} \frac{\boldsymbol{f}_{t} \otimes \boldsymbol{n}}{1+\boldsymbol{a}_{c}:(\boldsymbol{n} \otimes \boldsymbol{n})},
\end{aligned}
$$

from which two force anisotropy tensors are defined:

$\boldsymbol{a}_{n}=\frac{15}{2}\left(\frac{\boldsymbol{\phi}_{n}}{\operatorname{tr}\left(\boldsymbol{\phi}_{n}\right)}-\frac{1}{3} \boldsymbol{I}\right)$

$\boldsymbol{a}_{t}=\frac{15}{3} \frac{\phi_{t}}{\operatorname{tr}\left(\phi_{n}\right)}$

where $\operatorname{tr}(\cdot)$ is the trace operator. Equation (18) implies $\operatorname{tr}\left(\phi_{t}\right)=0$ given the normality of $\boldsymbol{t}$ and $\boldsymbol{n}$.

The deviatoric invariants of these tensors represent the degree of anisotropy:

$a_{[]}=\operatorname{sign}\left(S_{[]}\right) \sqrt{\frac{3}{2} \boldsymbol{a}_{[]}: \boldsymbol{a}_{[]}}$

where the subscript [] stands for $c, n$, or $t$, corresponding to the three aforementioned anisotropy tensors, respectively. $S_{[]}$is a normalized first joint invariant between $\mathbf{s}=\boldsymbol{\sigma}-p \boldsymbol{I}$ and each of the anisotropy tensors [19, 35], given by

$S_{[]}=\frac{\boldsymbol{a}_{[]}: \mathbf{s}}{\sqrt{\boldsymbol{a}_{[]}: \boldsymbol{a}_{[]}} \sqrt{\mathbf{s}: \mathbf{s}}}$

The well-known deviatoric stress is $q=\sqrt{(3 / 2) \mathbf{s}: \mathbf{s}}$. Generally, $S_{[]}$quantifies the level of coaxiality between two tensors, with $S_{[]}=1.0$ corresponding to the case where two tensors are proportional. $S_{[]}$can be regarded as defining the relative orientations of the principal axes (PA) of $\boldsymbol{a}_{[]}$with respect to that of $\mathbf{s}$, i.e., the level of coaxiality. Its sign is positive (respectively, negative) when the angle between principal axes is below (respectively, above) $\pi / 4$.

Figure 18 displays the evolutions of $S_{[]}$at the states where $\tau_{y} / p_{0}$ reaches $\pm \mathrm{CSR}_{y}$ in each loading cycle for the four shear paths. These are the points $\mathrm{C}_{1}$ and $\mathrm{C}_{3}$ of each loading cycle where the system forms a stable load-bearing network according to the previous analysis of particle connectivity. In each loading cycle, one expects marked oscillations of $S_{[]}$, which is indeed what we observe for the 1-D linear, 2-D linear, and 8-like tests as shown in the zoom-in window of each plot, but not for the circular test. The difficulty in dealing with cyclic loading is the absence of an ultimately converged state, such as the critical state (CS) for monotonic loading. For the conventional cyclic simple shear test, the closest states to the CS are $\mathrm{C}_{1}$ and $\mathrm{C}_{3}$ and thus assumed also for the other three shear paths. It is generally believed that $q / p$ reaches its critical state values 


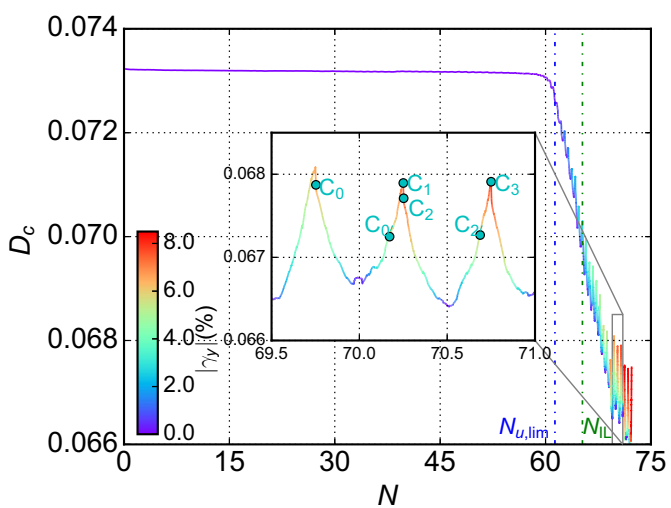

(a)

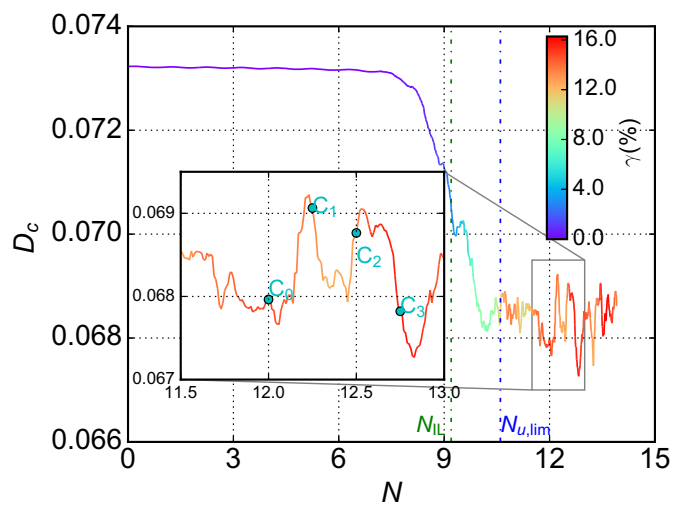

(c)

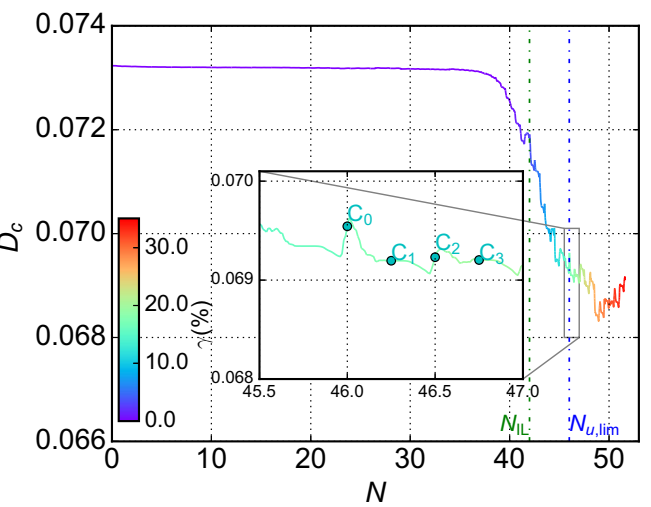

(b)

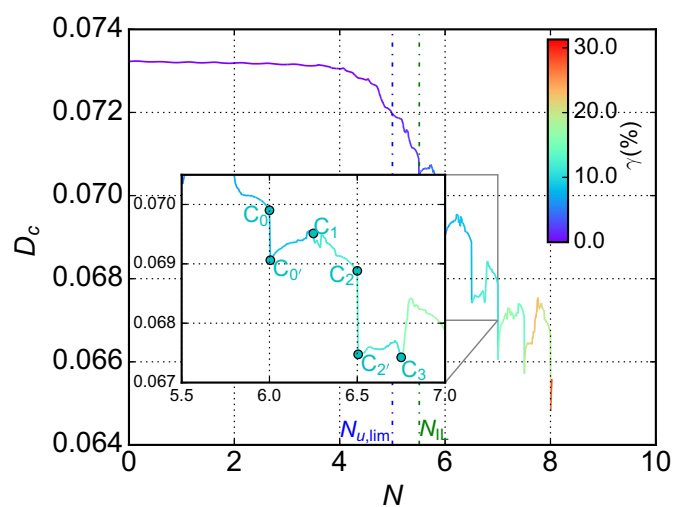

(d)

Fig. 17 Evolution of centroid distance $D_{c}$ for different shear paths: a 1-D linear; b 2-D linear; c circular; d 8-like

at these states after sufficient loading cycles. $S_{n}$ is always close to 1 , meaning that force anisotropy closely follows the stress deviator.

It is remarkable that the force anisotropy tensors $\boldsymbol{a}_{n}$ and $\boldsymbol{a}_{t}$ are nearly proportional to $\mathbf{s}$ from the beginning of cyclic loading at $\mathrm{C}_{1}$ and $\mathrm{C}_{3}$. In contrast, during the pre-liquefaction period they are not fully proportional although noncoaxiality decreases as the geometrical structure of the system changes until $\boldsymbol{a}_{c}$ becomes nearly proportional to $\mathbf{s}$ at the selected states. All the anisotropy tensors become nearly proportional to $\mathbf{s}$ at selected states in the post-liquefaction period. During the selected loading cycle in each inset, a significant change in $S_{[]}$is observed at the instance of unloading, where $S_{t}$ oscillates. In the subsequent loading, $\boldsymbol{a}_{t}$ and $\boldsymbol{a}_{n}$ adjust themselves to follow $\mathbf{s}$ more quickly than $\boldsymbol{a}_{c}$. It should be noted that the three anisotropy tensors in the case of circular shear path change almost at the same pace with $\mathbf{s} . S_{n}$ and $S_{c}$ become negative only in the 1-D linear path upon unjamming, whereas only $S_{t}$ becomes negative in the 8 -like path. Note that the limit $r_{u} \simeq 1$ is reached only in these two paths.
According to $[48,55]$, the shear strength normalized by the mean stress is with a good approximation a linear combination of the three anisotropy tensors:

$\frac{\mathbf{s}}{p} \simeq \frac{2}{5}\left(\boldsymbol{a}_{c}+\boldsymbol{a}_{n}+\frac{3}{2} \boldsymbol{a}_{t}\right)$

Since these anisotropy tensors are nearly coaxial with the stress tensor up to very short deviations during unjamming, as observed in Fig. 18, at selected states after sufficient number of loading cycles, Eq. (23) can be further simplified as

$\frac{q}{p} \simeq \frac{2}{5}\left(a_{c}+a_{n}+\frac{3}{2} a_{t}\right)$

This equation is checked for the four shear paths in Figs. 19a, c, e and g, where the evolutions of fabric and force anisotropies at $\mathrm{C}_{1}$ and $\mathrm{C}_{3}$ of each loading cycle are shown along with a zoom-in window displaying the detailed change of anisotropies during the selected postliquefaction loading cycles. In the post-liquefaction period, the three anisotropies tend to level off, with values increasing in the order of $a_{t}, a_{c}$, and $a_{n}$.

Figure 19b, d, f, and $\mathrm{h}$ shows the anisotropies and deviatoric stress ratio $q / p$, as well as its approximation 


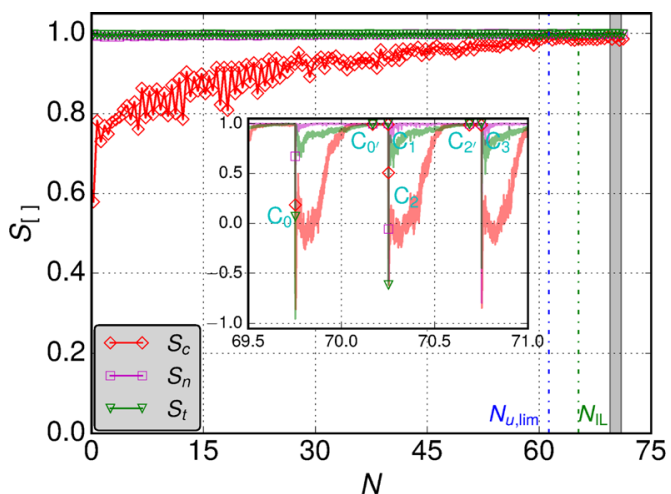

(a)

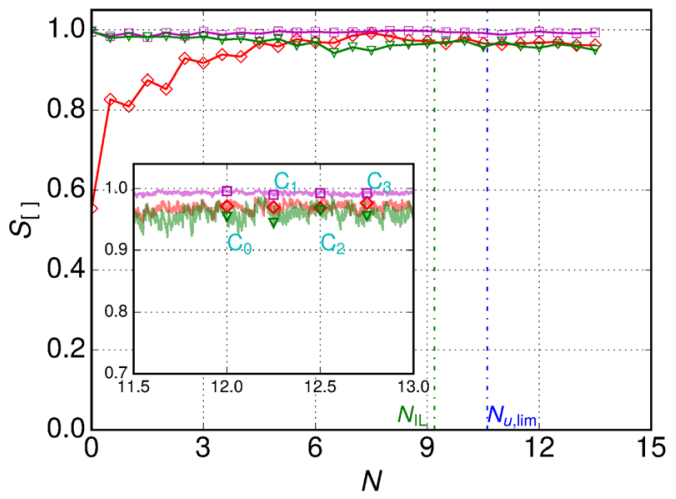

(c)

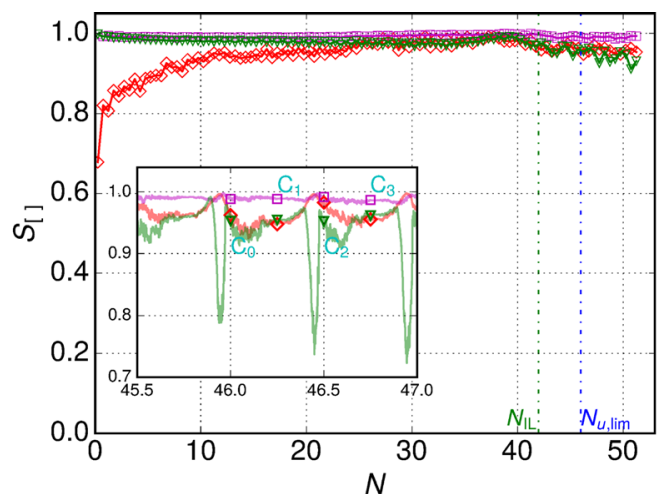

(b)

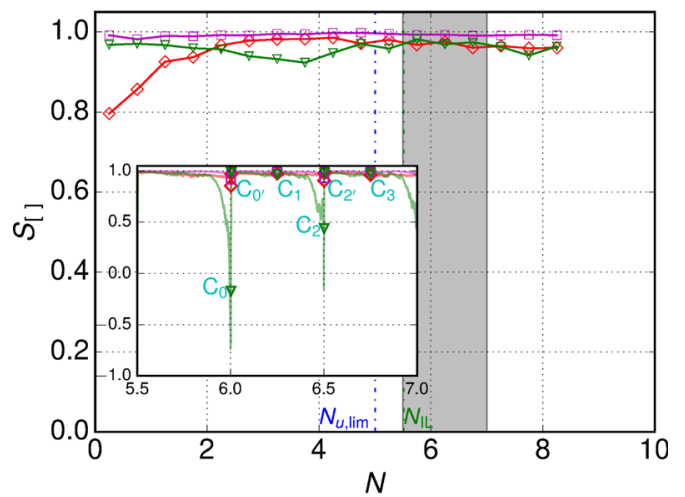

(d)

Fig. 18 Evolution of the normalized first joint invariant between fabric tensors and deviatoric stress tensor at $C_{1}$ and $C_{3}$ of each loading cycle for different shear paths: a 1-D linear; b 2-D linear; c circular; $\mathbf{d}$ 8-like. The insets zoom in on a few cycles in the marked window

$(2 / 5) a_{c}+(2 / 5) a_{n}+(3 / 5) a_{t}$ by Eq. (24). We see that this approximation holds quite nicely during the whole cyclic shearing. We also see how much each anisotropy additively contributes to $q / p$. After the initial liquefaction, the weighted contribution of each anisotropy to $q / p$ reaches a plateau where we have $a_{c} \simeq 0.4, a_{n} \simeq 0.5$ and $a_{t} \simeq 0.1$, irrespective of the shear path type. One may attribute this universal partition to the intrinsic feature of the material and its state, which can only change with particle shape and size polydispersity based on studies of monotonic loading $[8,45]$.

\section{Conclusion}

In this paper, we used a 3D DEM to conduct a comprehensive series of multidirectional cyclic shear tests for 1-D linear, 2-D linear, circular/oval, and 8-like paths, all under constant volume condition. At the system scale, the accumulation of large shear strain was observed for all the simulated tests, although the mean stress does not vanish for some of them. A well-defined relationship was shown between the limit pore pressure ratio and the corresponding shear stress ratio. With initial liquefaction defined as the state of total shear strain reaching $3 \%$ for the first time, the cyclic liquefaction resistance was found to decrease in the order of 1-D linear, 2-D linear, circular, and 8-like, for $\mathrm{AR}=\mathrm{CSR}_{x} / \mathrm{CSR}_{y}=1$ in the two latter cases. As $\mathrm{AR}$ decreases with fixed value of $\operatorname{CSR}_{y}$, both circular/oval and 8-like paths present enhanced cyclic resistance, which is expected to approach the value of 1-D linear path as $\mathrm{AR} \rightarrow 0$. As to the effect of $\mathrm{SSR}_{[]}$, for all four shear paths the cyclic liquefaction resistance declines first with increasing $\mathrm{SSR}_{[]}$and then increases later for larger values of $\mathrm{SSR}_{[]}$. A subsequent decrease in cyclic resistance is observed only for circular paths.

A detailed micromechanical analysis was also carried out to investigate three aspects. From the viewpoint of system stability, the coordination number and the particle connectivity diagram indicate that the system becomes transiently under-constrained for the 1-D linear and 8-like shear paths, occurring at the instance of vanishing mean stress, whereas the system stays over-constrained in the 2D linear and circular shear paths. The system's underconstrained state is also characterized by a large proportion of mobilized contacts and a scattering of short and weak 


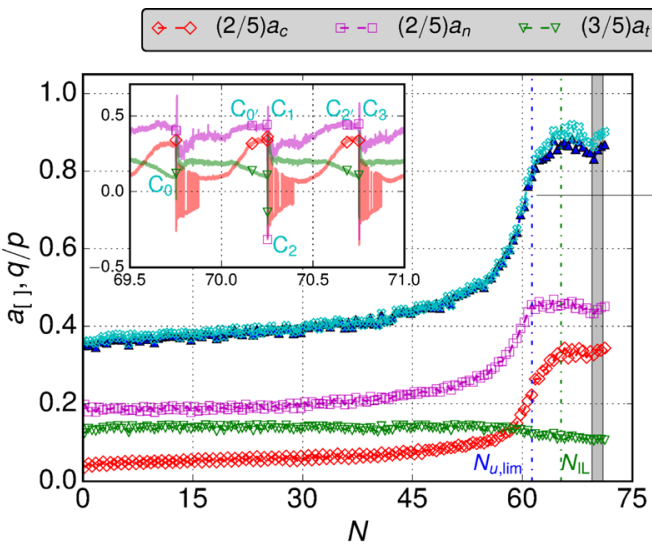

(a)

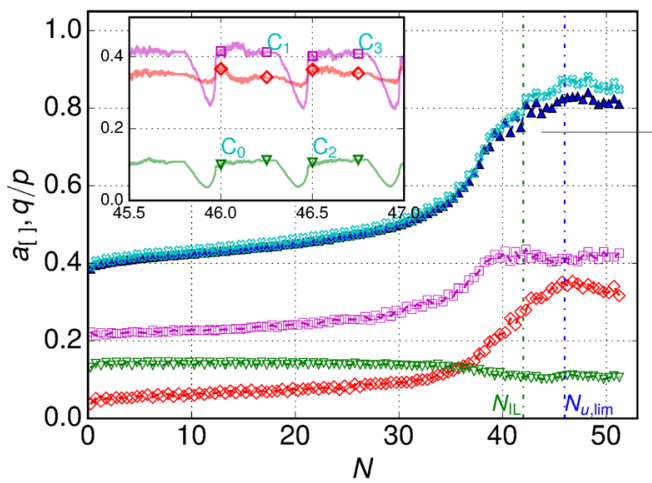

(c)

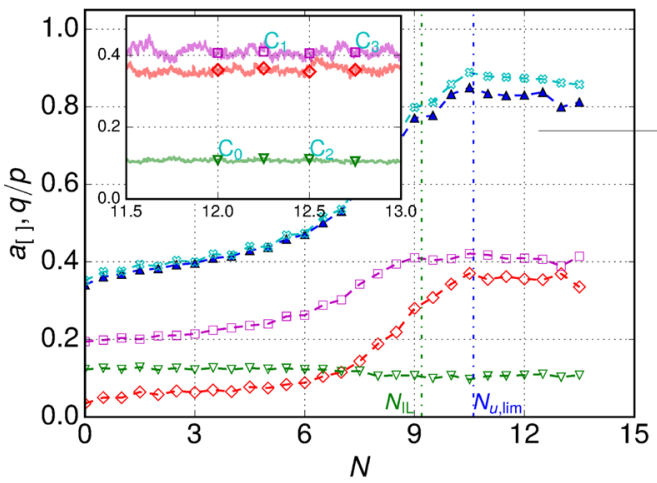

(e)

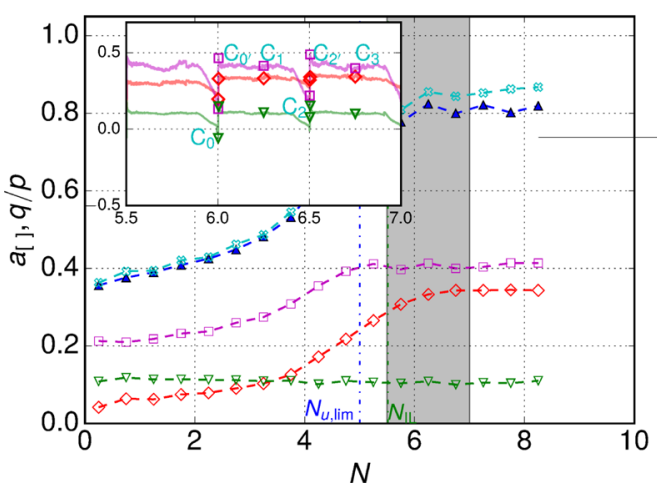

(g)

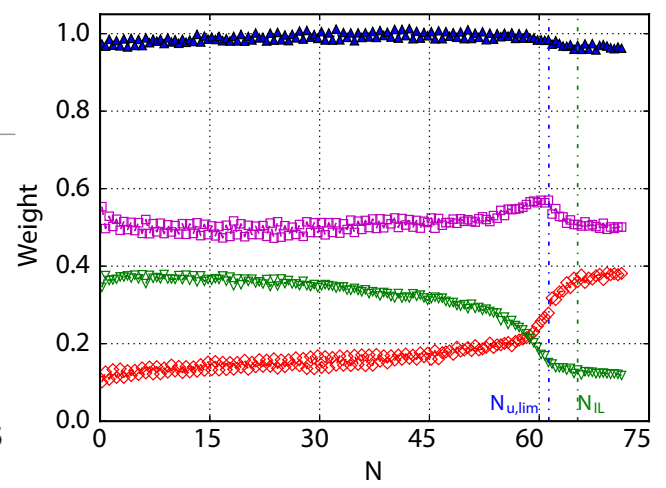

(b)

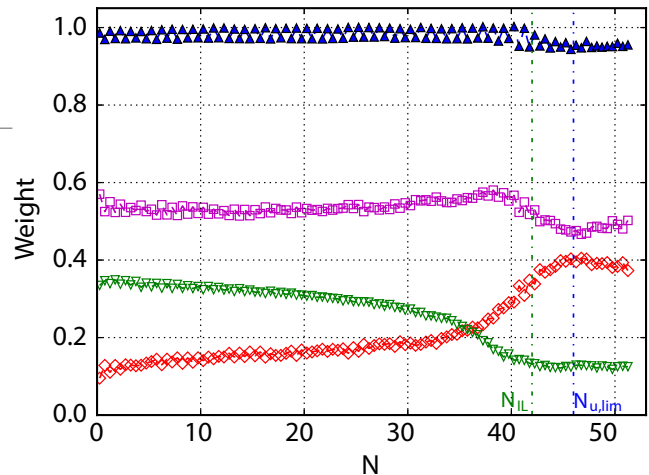

(d)

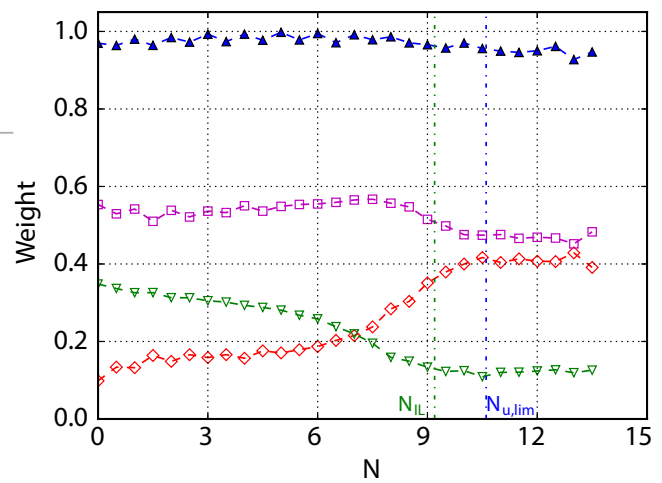

(f)

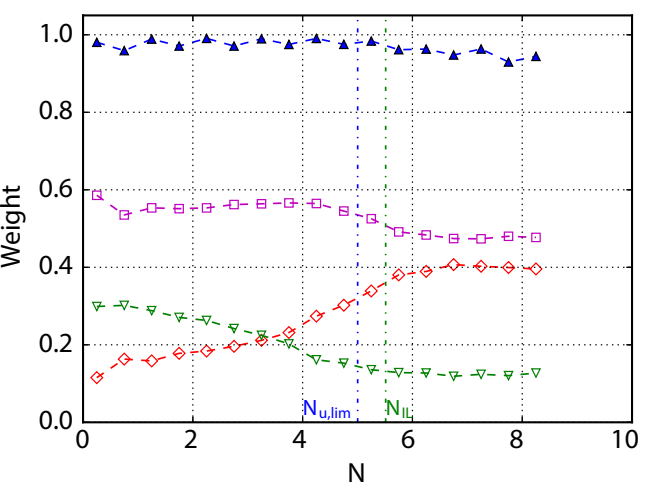

(h) 
4Fig. 19 Evolution of the anisotropies at $\mathrm{C}_{1}$ and $\mathrm{C}_{3}$ of each loading cycle and their weights contributing to deviatoric stress ratio $q$ / $p$ according to Eq. (24) for shear path of a, b 1-D linear; c, d 2-D linear; e, f circular; $\mathbf{g}, \mathbf{h}$ 8-like

force chains. A particle-void fabric indicator called centroid distance $D_{c}$ was adopted to analyze the shear strain development. A significant drop of $D_{c}$ occurs near the initial liquefaction, indicating a noticeable redistribution of voids by destroying large voids into small ones. $D_{c}$ presents a general decreasing trend in the post-liquefaction period due to the accumulation of shear strain. In the 1-D linear and 8-like tests, the change of $D_{c}$ mainly happens when $r_{u} \geq 0.99$, corresponding to the deformation mode of cyclic mobility. In the 2-D linear and circular tests, the change of $D_{c}$ occurs randomly in the whole loading cycle, corresponding to a deformation mode of residual deformation. Finally, the fabric and force anisotropies were calculated to explain the source of the load-bearing network where force anisotropy tensors quickly become almost coaxial with the deviatoric stress tensor while fabric anisotropy tensor needs most of the pre-liquefaction period to follow the loading. The weighted sum of these anisotropies can well approximate the deviatoric stress ratio at the peak of applied shear stress; their contributions to the post-liquefaction period level off, with the values that are independent of the shear path type.

The observed differences in the long-time behavior depending on the shear path show that the simple shear tests are insufficient to characterize the rheological behavior of granular materials under cyclic shearing. In particular, the liquefaction in the sense of large shear strains does not require the vanishing of the mean stress. Large strains are obtained through a smaller number of cycles when combinations of shear stress happen in two directions. These effects have to do with how the evolution of the microstructure interacts with the subsequent change of the shear stress direction or sign, conducting the system through different paths toward liquefaction. This work can be pursued to further analyze the spatiotemporal behavior of cyclic shearing along different paths by including the effects of material parameters such as the sliding, rolling, and twisting coefficients of friction are considered as proxy parameters for particle shape variables. The sample void ratio is another basic parameter that directly impacts the liquefaction resistance and shear strain development. It is essential to formulate necessary and sufficient conditions for the onset of liquefaction from the particle-scale parameters such as the coordination number and the fabric and force anisotropies for a given void ratio and particle shape.
Acknowledgements Financial support for this study was provided by the Natural Sciences and Engineering Research Council of Canada (NSERC). M. Yang and M. Taiebat would like to thank Professor Y. Vaid for the insightful discussions that led to Fig. 9.

\section{Declarations}

Conflict of interest The authors declare that they have no conflict of interest.

\section{References}

1. Alonso-Marroquin F, Herrmann HJ (2004) Ratcheting of granular materials. Phys Rev Lett 92(5)

2. Alonso-Marroquin F, Luding S, Herrmann HJ, Vardoulakis I (2005) Role of anisotropy in the elastoplastic response of a polygonal packing. Phys Rev E 71(5)

3. Azéma E, Radjaï F (2012) Force chains and contact network topology in sheared packings of elongated particles. Phys Rev E 85(3)

4. Bi D, Zhang J, Chakraborty B, Behringer RP (2011) Jamming by shear. Nature 480(7377):355-358

5. Bonilla RRO (2004) Numerical simulations of undrained granular media. Ph.D. thesis, University of Waterloo, University of Waterloo Waterloo, Canada

6. Boulanger RW, Seed RB (1995) Liquefaction of sand under bidirectional monotonic and cyclic loading. J Geotech Eng 121(12):870-878

7. Boulanger RW, Seed RB, Chan CK, Seed HB, Sousa J (1991) Liquefaction behavior of saturated sands under uni-directional and bi-directional monotonic and cyclic simple shear loading. Tech. Rep. 2, University of California, Berkeley, The address of the publisher. UCB/GT/91-08

8. Cantor D, Azéma E, Sornay P, Radjaï F (2018) Rheology and structure of polydisperse three-dimensional packings of spheres. Phys Rev E 98(5)

9. Carlton BD, Kaynia AM (2016) Comparison of the seismic response of offshore slopes using 1,2, or 3 ground motion components. Offshore Technol Conf. Houston, TX, pp 1-12

10. Cerna-Diaz A, Olson SM, Numanoglu OA, Hashash YMA, Bhaumik L, Rutherford CJ, Weaver T (2017) Free-field cyclic response of dense sands in dynamic centrifuge tests with 1D and 2D shaking. In: Geotechnical Frontiers 2017, pp. 121-130. American Society of Civil Engineers

11. Chiaro G, Koseki J, Sato T (2012) Effects of initial static shear on liquefaction and large deformation properties of loose saturated Toyoura sand in undrained cyclic torsional shear tests. Soils Found 52(3):498-510

12. Cundall PA, Strack ODL (1979) A discrete numerical model for granular assemblies. Géotechnique 29(1):47-65

13. Dyvik R, Lacasse S, Berre T, Raadim B (1987) Comparison of truly undrained and constant volume direct simple shear tests. Géotechnique 37(1):3-10

14. El Shafee O, Abdoun TH, Zeghal M (2017) Centrifuge modelling and analysis of site liquefaction subjected to biaxial dynamic excitations. Géotechnique 67(3):260-271

15. Estrada N, Azéma E, Radjaï F, Taboada A (2011) Identification of rolling resistance as a shape parameter in sheared granular media. Phys Rev E 84(1)

16. Evans TM, Zhang L (2019) A numerical study of particle friction and initial state effects on the liquefaction of granular assemblies. Soil Dynamics and Earthquake Engineering 126 
17. Ghaboussi J, Dikmen SU (1981) Liquefaction analysis for multidirectional shaking. J Geotech Eng Div 107(5):605-627

18. Gong G, Thornton C, Chan AHC (2012) DEM simulations of undrained triaxial behavior of granular material. J Eng Mech 138(6):560-566

19. Guo N, Zhao J (2013) The signature of shear-induced anisotropy in granular media. Comput Geotech 47:1-15

20. Hall SA, Bornert M, Desrues J, Pannier Y, Lenoir N, Viggiani G, Bésuelle P (2010) Discrete and continuum analysis of localised deformation in sand using $\mathrm{x}$-ray $\mu \mathrm{ct}$ and volumetric digital image correlation. Géotechnique 60(5):315-322

21. Huang X, Hanley KJ, Zhang Z, Kwok C, Xu M (2019) Jamming analysis on the behaviours of liquefied sand and virgin sand subject to monotonic undrained shearing. Comput Geotech 111:112-125

22. Huang X, Hanley KJ, Zhang Z, Kwok CY (2019) Structural degradation of sands during cyclic liquefaction: insight from DEM simulations. Comput Geotech 114

23. Huang X, Kwok CY, Hanley KJ, Zhang Z (2018) Dem analysis of the onset of flow deformation of sands: linking monotonic and cyclic undrained behaviours. Acta Geotech 13(5):1061-1074

24. Hyodo M, Murata H, Yasufuku N, Fujii T (1991) Undrained cyclic shear strength and residual shear strain of saturated sand by cyclic triaxial tests. Soils Found 31(3):60-76

25. Idriss IM, Boulanger RW (2006) Semi-empirical procedures for evaluating liquefaction potential during earthquakes. Soil Dyn Earthq Eng 26(2-4):115-130

26. Ishihara K, Yamazaki F (1980) Cyclic simple shear tests on saturated sand in multi-directional loading. Soils Found 20(1):45-59

27. Jiang M, Zhang A, Li T (2019) Distinct element analysis of the microstructure evolution in granular soils under cyclic loading. Granular Matter 21(2):39

28. Kammerer AM, Pestana JM, Seed RB (2002) Undrained response of monterey $0 / 30$ sand under multidirectional cyclic simple shear loading conditions. Geotechnical Engineering Report UCB/GT/ 02-01, University of California, Berkeley

29. Kammerer AM, Pestana JM, Seed RB (2005) Behavior of monterey $0 / 30$ sand under multidirectional loading conditions. In: Geomechanics: testing, modeling, and simulation, pp. 154-173. American Society of Civil Engineers

30. Kanatani KI (1984) Distribution of directional data and fabric tensors. Int J Eng Sci 22(2):149-164

31. Kruyt NP (2010) Micromechanical study of plasticity of granular materials. Comptes rendus mécanique 338(10-11):596-603

32. Kuhn MR (2017) Granular geomechanics. Elsevier

33. Kuhn MR, Daouadji A (2019) Stress fluctuations during monotonic loading of dense three-dimensional granular materials. Granular Matter 21(1): 10

34. Kuhn MR, Renken HE, Mixsell AD, Kramer SL (2014) Investigation of cyclic liquefaction with discrete element simulations. J Geotech Geoenviron Eng 140(12):04014075

35. Li XS, Dafalias YF (2012) Anisotropic critical state theory: role of fabric. J Eng Mech 138(3):263-275

36. Luding S (2008) Cohesive, frictional powders: contact models for tension. Granular Matter 10(4):235-246

37. Majmudar TS, Behringer RP (2005) Contact force measurements and stress-induced anisotropy in granular materials. Nature 435(7045):1079-1082

38. Martin EL, Thornton C, Utili S (2020) Micromechanical investigation of liquefaction of granular media by cyclic 3D DEM tests. Géotechnique 70(10):906-915

39. Matsuda H, Hendrawan AP, Ishikura R, Kawahara S (2011) Effective stress change and post-earthquake settlement properties of granular materials subjected to multi-directional cyclic simple shear. Soils Found 51(5):873-884
40. MiDi GDR (2004) On dense granular flows. Eur Phys J E 14(4):341-365

41. Morimoto T, Otsubo M, Koseki J (2021) Microscopic investigation into liquefaction resistance of pre-sheared sand: effects of particle shape and initial anisotropy. Soils Found

42. Mutabaruka P (2013) Numerical modeling of immersed granular media: initiation and propagation of avalanches in a fluid. Ph.D. thesis, Université Montpellier II-Science and Technology of Languedoc, Montpellier, France

43. Mutabaruka P, Taiebat M, Pellenq RJM, Radjaï F (2019) Effects of size polydispersity on random close-packed configurations of spherical particles. Phys Rev E 100(4)

44. Ng TT, Dobry R (1994) Numerical simulations of monotonic and cyclic loading of granular soil. J Geotech Eng 120(2):388-403

45. Nguyen DH, Azéma E, Sornay P, Radjaï F (2015) Effects of shape and size polydispersity on strength properties of granular materials. Phys Rev E 91(3)

46. Oda M (1982) Fabric tensor for discontinuous geological materials. Soils Found 22(4):96-108

47. O'Sullivan C (2011) Particulate discrete element modelling: a geomechanics perspective. CRC Press

48. Ouadfel H, Rothenburg L (2001) 'Stress-force-fabric' relationship for assemblies of ellipsoids. Mech Mater 33(4):201-221

49. Pouragha M, Wan R (2016) Onset of structural evolution in granular materials as a redundancy problem. Granular Matter 18(3):38

50. Pyke RM, Seed RB, Chan CK (1975) Settlement of sands under multidirectional shaking. J Geotech Eng Div 101(4):379-398

51. Radjaï F, Dubois F (2011) Discrete-element modeling of granular materials. Wiley-Iste, UK

52. Radjai F, Richefeu V (2009) Bond anisotropy and cohesion of wet granular materials. Philos Trans R Soc A: Math Phys Eng Sci 367(1909):5123-5138

53. Rahman MM, Nguyen HBK, Fourie AB, Kuhn MR (2021) Critical state soil mechanics for cyclic liquefaction and postliquefaction behavior: DEM study. J Geotech Geoenviron Eng 147(2):04020166

54. Reyes A, Adinata J, Taiebat M (2019) Impact of bidirectional seismic shearing on the volumetric response of sand deposits. Soil Dyn Earthq Eng 125

55. Rothenburg L, Bathurst RJ (1989) Analytical study of induced anisotropy in idealized granular materials. Géotechnique 39(4):601-614

56. Satake M (1982) Fabric tensor in granular materials. In: Vermeer PA, Lager HJ (eds) Deformation and failure of granular materials. Balkema, Rotterdan, pp 63-68

57. Schwager T, Pöschel $T$ (2007) Coefficient of restitution and linear-dashpot model revisited. Granular Matter 9(6):465-469

58. Sitharam TG (2003) Discrete element modelling of cyclic behaviour of granular materials. Geotech Geol Eng 21(4):297-329

59. Sitharam TG, Vinod JS, Ravishankar BV (2009) Post-liquefaction undrained monotonic behaviour of sands: experiments and dem simulations. Géotechnique 59(9):739-749

60. Soroush A, Ferdowsi B (2011) Three dimensional discrete element modeling of granular media under cyclic constant volume loading: a micromechanical perspective. Powder Technol 212(1):1-16

61. Su D, Li XS (2008) Impact of multidirectional shaking on liquefaction potential of level sand deposits. Géotechnique 58(4):259-267

62. Sun M (2019) The effects of multidirectional loading on soil liquefaction. Ph.D. thesis, University of Cambridge

63. Thornton C (2000) Numerical simulations of deviatoric shear deformation of granular media. Géotechnique 50(1):43-53

64. Thornton C (2015) Granular dynamics, contact mechanics and particle system simulations. Springer, Berlin 
65. Vaid YP, Chern JC (1983) Effects of static shear on resistance to liquefaction. Soils Found 23(1):47-60

66. Vaid YP, Stedman JD, Sivathayalan S (2001) Confining stress and static shear effects in cyclic liquefaction. Can Geotech J 38(3):580-591

67. Voivret C, Radjaï F, Delenne JY, Youssoufi MSE (2007) Spacefilling properties of polydisperse granular media. Phys Rev E $76(2)$

68. Wang G, Wei J (2016) Microstructure evolution of granular soils in cyclic mobility and post-liquefaction process. Granular Matter 18(3):51

69. Wang R, Fu P, Zhang JM, Dafalias YF (2016) DEM study of fabric features governing undrained post-liquefaction shear deformation of sand. Acta Geotech 11(6):1321-1337

70. Wang R, Fu P, Zhang JM, Dafalias YF (2019) Fabric characteristics and processes influencing the liquefaction and re-liquefaction of sand. Soil Dyn Earthq Eng 125

71. Wei J (2017) Discrete element analysis of fabric evolution in cyclic liquefaction of granular soils. Ph.D. thesis, The Hong Kong University of Science and Technology, Hong Kong

72. Wei J, Huang D, Wang G (2018) Microscale descriptors for particle-void distribution and jamming transition in pre-and postliquefaction of granular soils. J Eng Mech 144(8):04018067

73. Wei J, Huang D, Wang G (2020) Fabric evolution of granular soils under multidirectional cyclic loading. Acta Geotechnica pp $1-15$

74. Wei J, Wang G (2017) Discrete-element method analysis of initial fabric effects on pre-and post-liquefaction behavior of sands. Géotech Lett 7(2):161-166
75. Wichtmann T, Triantafyllidis T (2016) An experimental database for the development, calibration and verification of constitutive models for sand with focus to cyclic loading: part i-tests with monotonic loading and stress cycles. Acta Geotech 11(4):739-761

76. Yang J, Sze HY (2011) Cyclic behaviour and resistance of saturated sand under non-symmetrical loading conditions. Géotechnique 61(1):59-73

77. Yang M, Seidalinov G, Taiebat M (2019) Multidirectional cyclic shearing of clays and sands: Evaluation of two bounding surface plasticity models. Soil Dyn Earthq Eng 124:230-258

78. Yang M, Taiebat M, Vaid YP (2016) Bidirectional monotonic and cyclic shear testing of soils: state of knowledge. In: 69th Canadian geotechnical conference. Vancouver, BC, Canada. Paper ID: 4198, 8 pages

79. Zeghal M, El-Shafee O, Abdoun T (2018) Analysis of soil liquefaction using centrifuge tests of a site subjected to biaxial shaking. Soil Dyn Earthq Eng 114:229-241

80. Zhang L, Evans TM (2020) Investigation of initial static shear stress effects on liquefaction resistance using discrete element method simulations. Int J Geomech 20(7):04020087

81. Zhou W, Liu J, Ma G, Chang X (2017) Three-dimensional DEM investigation of critical state and dilatancy behaviors of granular materials. Acta Geotech 12(3):527-540 